\title{
THE COLORED JONES POLYNOMIAL, THE CHERN-SIMONS INVARIANT, AND THE REIDEMEISTER TORSION OF THE FIGURE-EIGHT KNOT
}

\author{
HITOSHI MURAKAMI \\ Dedicated to the memory of my father, Akira Murakami (1930-2011)
}

\begin{abstract}
We show that from the asymptotic behavior of an evaluation of the colored Jones polynomial of the figure-eight knot we can extract the ChernSimons invariant and the twisted Reidemeister torsion associated with a representation of the fundamental group of the knot complement to the twodimensional complex special linear group.
\end{abstract}

\section{INTRODUCTION}

Let $J_{N}(K ; q)$ be the colored Jones polynomial of a knot $K$ in the three-sphere $S^{3}$ associated with the $N$-dimensional irreducible representation of $\mathfrak{s l}(2 ; \mathbb{C})$. We normalize it so that $J_{N}($ unknot; $q)=1$. Note that $J_{2}(K ; q)$ is the celebrated Jones polynomial 11] after a suitable change of variable.

In 1995 Kashaev introduced a complex valued knot invariant for each natural number $N$ by using quantum dilogarithm [12] and observed that its asymptotic behavior for large $N$ determines the hyperbolic volume for several hyperbolic knots [13. He also conjectured that it is also true for any hyperbolic knot. Here a knot is called hyperbolic if its complement has a unique complete hyperbolic structure with finite volume. It is proved in 2001 by J. Murakami and the author that his invariant coincides with $J_{N}(K ; \exp (2 \pi \sqrt{-1} / N))$ [21. We also generalized Kashaev's conjecture to the following volume conjecture.

Conjecture 1.1 (Volume Conjecture [13, 21]). Let $K$ be a knot in $S^{3}$ and $\operatorname{Vol}(K)$ denote the simplicial volume of $S^{3} \backslash K$. Then the following equality would hold:

$$
\lim _{N \rightarrow \infty} \frac{\log \left|J_{N}(K ; \exp (2 \pi \sqrt{-1} / N))\right|}{N}=\frac{\operatorname{Vol}(K)}{2 \pi} .
$$

See for example 20] about recent developments of the conjecture and its generalizations.

As one of the generalizations Yokota and the author [22] proved that for the figure-eight knot the colored Jones polynomial knows much more. Actually we showed that if we perturb the parameter $2 \pi \sqrt{-1}$ a little the corresponding limit determines the $\mathrm{SL}(2 ; \mathbb{C})$ Chern-Simons invariant associated with an irreducible representation of $\pi_{1}\left(S^{3} \backslash K\right)$ to $\mathrm{SL}(2 ; \mathbb{C})$ in the sense of Kirk and Klassen [14. In fact we showed the following theorem.

Date: November 3, 2018.

2000 Mathematics Subject Classification. Primary 57M27 57M25 57M50.

Key words and phrases. colored Jones polynomial, volume conjecture, Chern-Simons invariant, Reidemeister torsion.

The authors are supported by Grant-in-Aid for Challenging Exploratory Research (21654053). 
Theorem 1.2 ([22]). Let $E$ be the figure-eight knot. There exists a neighborhood $U \subset \mathbb{C}$ of 0 such that if $u \in(U \backslash \pi \sqrt{-1} \mathbb{Q}) \cup\{0\}$ then the following limit exists:

$$
\lim _{N \rightarrow \infty} \frac{\log J_{N}(K ; \exp ((u+2 \pi \sqrt{-1} / N)))}{N} .
$$

Moreover the limit determines the $\mathrm{SL}(2 ; \mathbb{C})$ Chern-Simons invariant associated with an irreducible representation of $\pi_{1}\left(S^{3} \backslash E\right)$ to $\mathrm{SL}(2 ; \mathbb{C})$ which is determined by the parameter $u$.

On the other hand, Andersen and Hansen [1, Theorem 1] refined the volume conjecture for the figure-eight knot as follows.

Theorem 1.3 ([1]). The following asymptotic equivalence holds:

$$
\begin{aligned}
& J_{N}(E ; \exp (2 \pi \sqrt{-1} / N)) \\
& \underset{N \rightarrow \infty}{\sim} \frac{1}{3^{1 / 4}} N^{3 / 2} \exp \left(\frac{N \operatorname{Vol}(E)}{2 \pi}\right) \\
&=2 \pi^{3 / 2}\left(\frac{2}{\sqrt{-3}}\right)^{1 / 2}\left(\frac{N}{2 \pi \sqrt{-1}}\right)^{3 / 2} \exp \left(\frac{N}{2 \pi \sqrt{-1}} \times \sqrt{-1} \operatorname{Vol}(E)\right) .
\end{aligned}
$$

Note that the twisted Reidemeister torsion and the Chern-Simons invariant associated with the unique complete hyperbolic structure of $S^{3} \backslash E$ are $2 / \sqrt{-3}$ and $\sqrt{-1} \operatorname{Vol}(E)$ respectively.

Note that we write $f(N) \underset{N \rightarrow \infty}{\sim} g(N)$ if and only if $\lim _{N \rightarrow \infty} f(N) / g(N)=1$ and that (1.1) follows from the equivalence relation above when $K$ is the figure-eight knot.

In this paper we refine Theorem 1.2 as Theorem 1.3 for the case where $u$ is real.

Theorem 1.4. Let $u$ be a real number with $0<u<\log ((3+\sqrt{5}) / 2)=0.9624 \ldots$ and put $\xi:=2 \pi \sqrt{-1}+u$. Then we have the following asymptotic equivalence of the colored Jones polynomial of the figure-eight knot E:

$$
J_{N}(E ; \exp (\xi / N)) \underset{N \rightarrow \infty}{\sim} \frac{\sqrt{-\pi}}{2 \sinh (u / 2)} T(u)^{1 / 2}\left(\frac{N}{\xi}\right)^{1 / 2} \exp \left(\frac{N}{\xi} S(u)\right),
$$

where

and

$$
S(u):=\operatorname{Li}_{2}\left(e^{u-\varphi(u)}\right)-\operatorname{Li}_{2}\left(e^{u+\varphi(u)}\right)-u \varphi(u)
$$

$$
T(u):=\frac{2}{\sqrt{\left(e^{u}+e^{-u}+1\right)\left(e^{u}+e^{-u}-3\right)}} .
$$

Here $\varphi(u):=\operatorname{arccosh}(\cosh (u)-1 / 2)$ and

$$
\operatorname{Li}_{2}(z):=-\int_{0}^{z} \frac{\log (1-x)}{x} d x
$$

is the dilogarithm function.

Note that $S(u)$ defines the $\operatorname{SL}(2 ; \mathbb{C})$ Chern-Simons invariant and $T(u)$ is the cohomological twisted Reidemeister torsion, both of which are associated with an irreducible representation of $\pi_{1}\left(S^{3} \backslash E\right)$ into $S L(2 ; \mathbb{C})$ sending the meridian to an element with eigenvalues $\exp (u / 2)$ and $\exp (-u / 2)$. See Section 4 for details.

Remark 1.5. Since the figure-eight knot is amphicheiral, we have $J_{N}\left(E ; q^{-1}\right)=$ $J_{N}(E ; q)$. Thus if $u<0$ we have

$$
\begin{aligned}
J_{N}(E ; \exp ((u+2 \pi \sqrt{-1}) / N)) & =\frac{J_{N}(E ; \exp ((-u-2 \pi \sqrt{-1}) / N))}{J_{N}(E ; \exp ((-u+2 \pi \sqrt{-1}) / N))},
\end{aligned}
$$


where $\bar{z}$ denotes the complex conjugate of $z$. So if we prove Theorem 1.4 for $u>0$, we have a similar asymptotic equivalence for $u<0$. Details are left to the readers.

Theorem 1.4 confirms the following conjecture in the case of the figure-eight knot for real $u$ with $0<u<\log ((3+\sqrt{5}) / 2)$.

Conjecture 1.6 ([8, 2]). Let $K$ be a hyperbolic knot. Then there exists a neighborhood $U \in \mathbb{C}$ of 0 such that if $u \in U \backslash \pi \sqrt{-1} \mathbb{Q}$, we have the following asymptotic equivalence:

$$
J_{N}(K ; \exp (\xi / N)) \underset{N \rightarrow \infty}{\sim} \frac{\sqrt{-\pi}}{2 \sinh (u / 2)} T(K ; u)^{1 / 2}\left(\frac{N}{\xi}\right)^{1 / 2} \exp \left(\frac{N}{\xi} S(K ; u)\right),
$$

where $\xi:=2 \pi \sqrt{-1}+u, T(K ; u)$ is the cohomological twisted Reidemeister torsion and $S(K ; u)$ is the $\mathrm{SL}(2 ; \mathbb{C})$ Chern-Simons invariant, both of which are associated with an irreducible representation of $\pi_{1}\left(S^{3} \backslash K\right)$ into $S L(2 ; \mathbb{C})$ sending the meridian to an element with eigenvalues $\exp (u / 2)$ and $\exp (-u / 2)$.

For physical interpretations of this conjecture, see for example [7, 2].

For torus knots we know the following result. Let $T(a, b)$ be the torus knot of type $(a, b)$ for positive coprime integers $a$ and $b$. It is known that the $\operatorname{SL}(2 ; \mathbb{C})$ character variety of $\pi_{1}\left(S^{3} \backslash T(a, b)\right)$ has $(a-1)(b-1) / 2$ components [15] (see also 18). Such components are indexed by a positive integer $k$ that is not a multiple of $a$ or $b$. See $[9, \S 2]$ for details. Let $\rho_{k}$ be an irreducible representation in the component indexed by $k, S_{k}(u)$ be the Chern-Simons invariant associated with $\rho_{k}$ with $\exp ( \pm u / 2)$ the eigenvalues of the image of the meridian by $\rho_{k}$, and $T_{k}$ be the cohomological twisted Reidemeister torsion associated with $\rho_{k}$. Then we have the following formulas [9]:

$$
S_{k}(u):=\frac{-(2 k \pi \sqrt{-1}-a b(2 \pi \sqrt{-1}+u))^{2}}{4 a b}
$$

and

$$
T_{k}:=\frac{16 \sin ^{2}(k \pi / a) \sin ^{2}(k \pi / b)}{a b} .
$$

Dubois and Kashaev [5], and Hikami and the author 9] obtain the following asymptotic equivalences.

Theorem $1.7(\underline{5]})$. For $u=0$ we have

$$
\begin{aligned}
J_{N}(T(a, b) ; \exp (2 \pi & \sqrt{-1} / N)) \\
& \sim \underset{N \rightarrow \infty}{\sim} \frac{\pi^{3 / 2}}{2 a b}\left(\frac{N}{2 \pi \sqrt{-1}}\right)^{3 / 2} \sum_{k=1}^{a b-1}(-1)^{k+1} k^{2} T_{k}^{1 / 2} \exp \left(\frac{N}{\xi} S_{k}(0)\right) .
\end{aligned}
$$

Note that since $T_{k}$ vanishes if a or $b$ divides $k$, the summation is for all the irreducible components of the character variety.

Theorem $1.8(9])$. Let $u$ be a complex number with $0<|u|<2 \pi /(a b)$. Then we have

when $\operatorname{Re} u>0$ and

$$
J_{N}(T(a, b) ; \exp (\xi / N)) \underset{N \rightarrow \infty}{\sim} \frac{1}{\Delta\left(T(a, b) ; e^{u}\right)}
$$

$$
\begin{aligned}
& J_{N}(T(a, b) ; \exp (\xi / N)) \\
& \quad \underset{N \rightarrow \infty}{\sim} \frac{1}{\Delta\left(T(a, b) ; e^{u}\right)}+\frac{\sqrt{-\pi}}{2 \sinh (u / 2)} \sum_{k=1}^{a b-1}(-1)^{k} T_{k}^{1 / 2}\left(\frac{N}{\xi}\right)^{1 / 2} \exp \left(\frac{N}{\xi} S_{k}(u)\right)
\end{aligned}
$$


when $\operatorname{Re} u<0$, where $\xi:=u+2 \pi \sqrt{-1}$ and $\Delta(T(a, b) ; t)$ is the Alexander polynomial. See [9] for more details.

The paper is organized as follows. In Section 2 we give an integral formula for the colored Jones polynomial using the quantum dilogarithm. We study its asymptotic behavior by using the saddle point method to give a proof of Theorem 1.4 in Section 3. In Section 4 we give topological interpretations of $S(u)$ and $T(u)$. Sections 5 to 8 are devoted to miscellaneous calculations.

\section{INTEGRAL FORMULA FOR THE COLORED JONES POLYNOMIAL}

In this section we use the quantum dilogarithm function to express the colored Jones polynomial of the figure-eight knot as an integral. We mainly follow [1].

First of all we recall the following formula due to Habiro and Le (see for example [17]).

$$
\begin{aligned}
J_{N}(E ; q) & =\sum_{k=0}^{N-1} \prod_{l=1}^{k}\left(q^{(N-l) / 2}-q^{-(N-l) / 2}\right)\left(q^{(N+l) / 2}-q^{-(N+l) / 2}\right) \\
& =\sum_{k=0}^{N-1} q^{-k N} \prod_{l=1}^{k}\left(1-q^{N-l}\right)\left(1-q^{N+l}\right) .
\end{aligned}
$$

For a complex number $\gamma$ with $\operatorname{Re}(\gamma)>0$, define the quantum dilogarithm $S_{\gamma}(z)$ as follows [6]:

$$
S_{\gamma}(z):=\exp \left(\frac{1}{4} \int_{C_{R}} \frac{e^{z t}}{\sinh (\pi t) \sinh (\gamma t)} \frac{d t}{t}\right),
$$

where $|\operatorname{Re}(z)|<\pi+\operatorname{Re}(\gamma)$ and $C_{R}$ is $(-\infty,-R] \cup \Omega_{R} \cup[R, \infty)$ with $\Omega_{R}:=$ $\{R \exp (\sqrt{-1}(\pi-s)) \mid 0 \leq s \leq \pi\}$ for $0<R<\min \{\pi /|\gamma|, 1\}$. Note that the poles of the integrand are $0, \pm \sqrt{-1}, \pm 2 \sqrt{-1}, \ldots$ and $\pm \pi \sqrt{-1} / \gamma, \pm 2 \pi \sqrt{-1} / \gamma, \ldots$

Remark 2.1. Note that in [1] it is assumed that $\gamma$ is real and $0<\gamma<1$ but we can define $S_{\gamma}(z)$ when $\operatorname{Re}(\gamma)>0$. See [3, (3.21)]. (Our quantum dilogarithm $S_{\gamma}(z)$ is equal to $\Phi(z /(2 \pi) ; \gamma / \pi)$ in [3].) We give a proof of the analyticity of $S_{\gamma}$ in Lemma 5.1

The following formula is well known and its proof can be found in [1, p. 530]. Note that they assume that $\gamma$ is real but their proof is also valid in our case.

Lemma 2.2. If $|\operatorname{Re}(z)|<\pi$, then we have

$$
\left(1+e^{\sqrt{-1} z}\right) S_{\gamma}(z+\gamma)=S_{\gamma}(z-\gamma)
$$

Putting $\gamma:=(2 \pi-\sqrt{-1} u) /(2 N)$ and $z:=\pi-\sqrt{-1} u-2 l \gamma(l=1,2, \ldots, N-1)$ in (2.2), we have

$$
\left(1+e^{\sqrt{-1}(\pi-\sqrt{-1} u-2 l \gamma)}\right) S_{\gamma}(\pi-\sqrt{-1} u-2 l \gamma+\gamma)=S_{\gamma}(\pi-\sqrt{-1} u-2 l \gamma-\gamma) .
$$

So for $k=1,2, \ldots, N-1$ we have

$$
\begin{aligned}
\prod_{l=1}^{k}(1-\exp ((N-l) \xi / N)) & =\prod_{l=1}^{k}\left(1+e^{\sqrt{-1}(\pi-\sqrt{-1} u-2 \pi l / N+\sqrt{-1} u l / N)}\right) \\
& =\prod_{l=1}^{k} \frac{S_{\gamma}(\pi-\sqrt{-1} u-(2 l+1) \gamma)}{S_{\gamma}(\pi-\sqrt{-1} u-(2 l-1) \gamma)} \\
& =\frac{S_{\gamma}(\pi-\sqrt{-1} u-(2 k+1) \gamma)}{S_{\gamma}(\pi-\sqrt{-1} u-\gamma)}
\end{aligned}
$$


Similarly we have

$$
\prod_{l=1}^{k}(1-\exp ((N+l) \xi / N))=\frac{S_{\gamma}(-\pi-\sqrt{-1} u+\gamma)}{S_{\gamma}(-\pi-\sqrt{-1} u+(2 k+1) \gamma)}
$$

Therefore we have

$$
\begin{aligned}
& J_{N}(E ; \exp (\xi / N)) \\
& \quad=\frac{S_{\gamma}(-\pi-\sqrt{-1} u+\gamma)}{S_{\gamma}(\pi-\sqrt{-1} u-\gamma)} \sum_{k=0}^{N-1} \exp (-k u) \frac{S_{\gamma}(\pi-\sqrt{-1} u-(2 k+1) \gamma)}{S_{\gamma}(-\pi-\sqrt{-1} u+(2 k+1) \gamma)} .
\end{aligned}
$$

Using $S_{\gamma}$ we define

$$
g_{N}(w):=\exp (-N u w) \frac{S_{\gamma}(\pi-\sqrt{-1} u+\sqrt{-1} \xi w)}{S_{\gamma}(-\pi-\sqrt{-1} u-\sqrt{-1} \xi w)} .
$$

Since $S_{\gamma}(z)$ is defined for $|\operatorname{Re}(z)|<\pi+\operatorname{Re}(\gamma)$, the function $g_{N}(w)$ is defined for $w$ with $|\pi-\operatorname{Im}(\xi w)|<\pi+\operatorname{Re}(\gamma)=\pi+\pi / N$, that is, $w$ is in the strip $-(2 \pi / u) \operatorname{Re}(w)-$ $\pi /(N u)<\operatorname{Im}(w)<-(2 \pi / u) \operatorname{Re}(w)+2 \pi / u+\pi /(N u)$ (Figure 1).

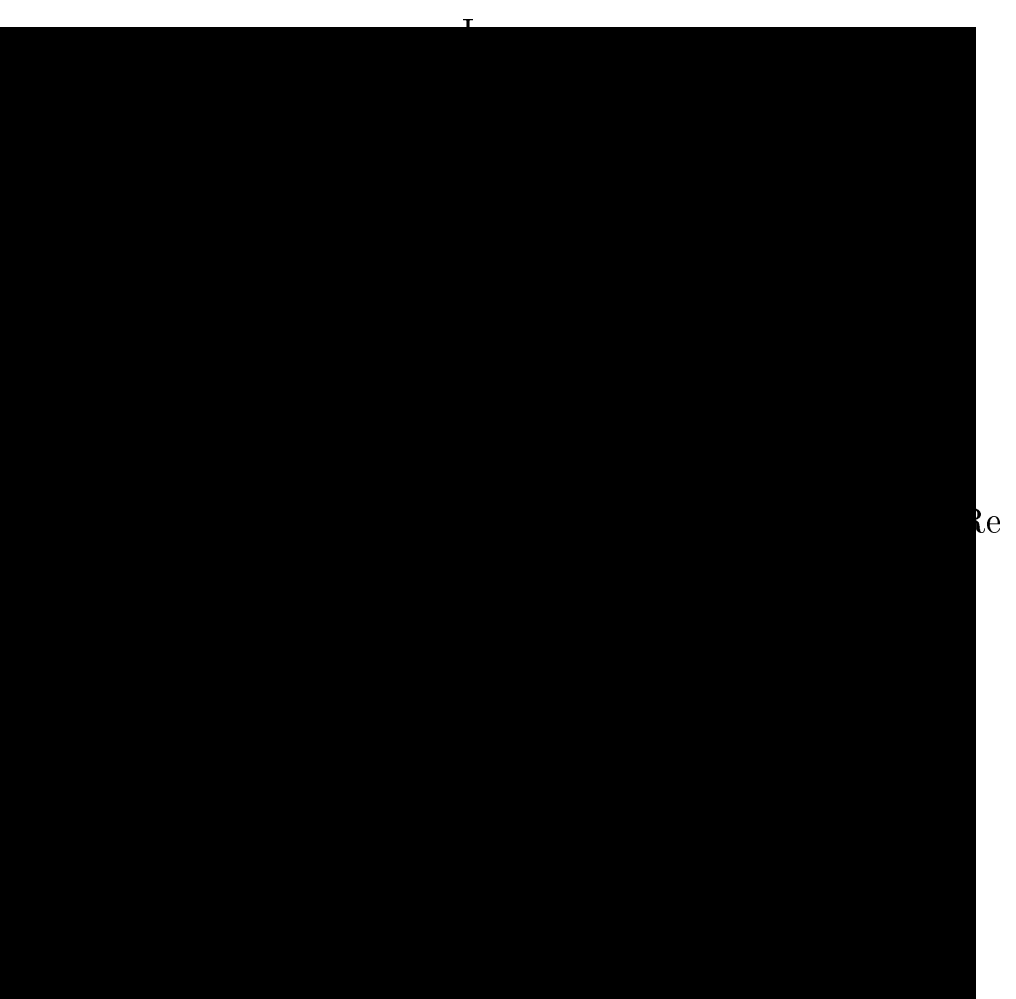

FiguRE 1. The function $g_{N}(\Phi$, respectively) is defined between the two dotted (thick, respectively) lines. The dashed parallelogram indicates the contour $C_{+}(\varepsilon) \cup C_{-}(\varepsilon)$.

For $0<\varepsilon<1 /(4 N)$, let $C_{+}(\varepsilon)$ be the polygonal line that connects $1-\varepsilon$, $1-u /(2 \pi)-\varepsilon+\sqrt{-1},-u /(2 \pi)+\varepsilon+\sqrt{-1}$, and $\varepsilon$, and $C_{-}(\varepsilon)$ be the polygonal line that connects $\varepsilon, u /(2 \pi)-\sqrt{-1}, 1+u /(2 \pi)-\sqrt{-1}$, and $1-\varepsilon$. See Figure 1. Put $C(\varepsilon):=C_{-}(\varepsilon) \cup C_{+}(\varepsilon)$. Note that the domain of $g_{N}(w)$ contains $C(\varepsilon)$.

By the residue theorem we have 


$$
\begin{aligned}
& J_{N}(E ; \exp (\xi / N)) \\
& \quad=\frac{S_{\gamma}(-\pi-\sqrt{-1} u+\gamma)}{S_{\gamma}(\pi-\sqrt{-1} u-\gamma)} \frac{\sqrt{-1} \exp (u / 2) N}{2} \int_{C(\varepsilon)} \tan (N \pi w) g_{N}(w) d w
\end{aligned}
$$

since the set of the poles of $\tan (N \pi w)$ inside $C(\varepsilon)$ is $\{(2 k+1) /(2 N) \mid k=$ $0,1,2, \ldots, N-1\}$ and the residue of each pole is $-1 /(N \pi)$.

Putting

$$
G_{ \pm}(N, \varepsilon):=\int_{C_{ \pm}(\varepsilon)} \tan (N \pi w) g_{N}(w) d w
$$

we have

$J_{N}(E ; \exp (\xi / N))=\frac{S_{\gamma}(-\pi-\sqrt{-1} u+\gamma)}{S_{\gamma}(\pi-\sqrt{-1} u-\gamma)} \frac{\sqrt{-1} \exp (u / 2) N}{2}\left(G_{+}(N, \varepsilon)+G_{-}(N, \varepsilon)\right)$.

\section{Approximating the integral Formula}

In this section we approximate the integral formula for the colored Jones polynomial obtained in the previous section.

Since $\tan (N \pi w)$ is close to $\sqrt{-1}(-\sqrt{-1}$, respectively) when $\operatorname{Im}(w)$ is positive and large (negative and $|\operatorname{Im}(w)|$ is large, respectively), we can approximate $G_{ \pm}(N, \varepsilon)$ by the integral of $g_{N}(w)$ on $C_{ \pm}(\varepsilon)$. In fact if we write

$$
G_{ \pm}(N, \varepsilon)= \pm \sqrt{-1} \int_{C_{ \pm}(\varepsilon)} g_{N}(w) d w+\int_{C_{ \pm}(\varepsilon)}(\tan (N \pi w) \mp \sqrt{-1}) g_{N}(w) d w
$$

then we have the following lemma.

Proposition 3.1 (see Equation (4.7) in [1]). There exists a positive constant $K_{1, \pm}$ independent of $N$ and $\varepsilon$ such that the following inequality holds:

$$
\left|\int_{C_{ \pm}(\varepsilon)}(\tan (N \pi w) \mp \sqrt{-1}) g_{N}(w) d w\right|<\frac{K_{1, \pm}}{N} .
$$

A proof is given in Section 6.

Now we approximate the integral of $g_{N}(w)$ along $C_{ \pm}(\varepsilon)$. Define

$$
\Phi(w):=\frac{1}{\xi}\left(\operatorname{Li}_{2}\left(e^{u-\xi w}\right)-\operatorname{Li}_{2}\left(e^{u+\xi w}\right)\right)-u w .
$$

Since $\mathrm{Li}_{2}$ is analytic in the region $\mathbb{C} \backslash(1, \infty)$, the function $\Phi$ is analytic in the region $\left\{w \in \mathbb{C} \mid-\frac{2 \pi}{u} \operatorname{Re}(w)<\operatorname{Im}(w)<-\frac{2 \pi}{u}(\operatorname{Re}(w)-1)\right\}$ (Figure 1).

Proposition 3.2 (see Equation (4.9) in [1]). Let $p(\varepsilon)$ be any contour in the parallelogram bounded by $C(\varepsilon)$ connecting $\varepsilon$ and $1-\varepsilon$, then there exists a positive constant $K_{2}$ independent of $N$ and $\varepsilon$ such that the following inequality holds.

$$
\left|\int_{p(\varepsilon)} g_{N}(w) d w-\int_{p(\varepsilon)} \exp (N \Phi(w)) d w\right| \leq \frac{K_{2} \log (N)}{N} \max _{w \in p(\varepsilon)}\{\exp (N \operatorname{Re} \Phi(w))\} .
$$

A proof is given in Section 7 .

We will study the asymptotic behavior of $\int_{C_{ \pm}(\varepsilon)} \exp (N \Phi(w)) d w$ for large $N$.

Since $\Phi(w)$ is analytic in the region $\left\{w \in \mathbb{C} \mid-\frac{2 \pi}{u} \operatorname{Re}(w)<\operatorname{Im}(w)<\right.$ $\left.-\frac{2 \pi}{u}(\operatorname{Re}(w)-1)\right\}$, we have

$$
\int_{C_{+}(\varepsilon)} \exp (N \Phi(w)) d w=\int_{C_{-}(\varepsilon)} \exp (N \Phi(w)) d w
$$

by Cauchy's integral theorem. 
We will apply the saddle point method (see for example [16, $\S 7.2]$ ) to approximate the integral $\int_{C_{-}(\varepsilon)} \exp (N \Phi(w)) d w$.

First we find a solution to the equation $d \Phi(w) / d w=0$. Since we have

$$
\frac{d \Phi(w)}{d w}=\log \left(1-e^{u-\xi w}\right)+\log \left(1-e^{u+\xi w}\right)-u
$$

a solution to the equation

$$
e^{\xi w}+e^{-\xi w}=e^{u}+e^{-u}-1
$$

can be a saddle point that we need. Put

$$
\begin{aligned}
\varphi(u) & :=\operatorname{arccosh}(\cosh (u)-1 / 2) \\
& =\log \left(\frac{1}{2}\left(e^{u}+e^{-u}-1-\sqrt{\left(e^{u}+e^{-u}+1\right)\left(e^{u}+e^{-u}-3\right)}\right)\right), \\
\tilde{\varphi}(u) & :=\varphi(u)+2 \pi \sqrt{-1}
\end{aligned}
$$

and

$$
w_{0}:=\frac{\tilde{\varphi}(u)}{\xi},
$$

where we choose the square root of $\left(e^{u}+e^{-u}+1\right)\left(e^{u}+e^{-u}-3\right)$ as a positive multiple of $\sqrt{-1}$ and the branch of $\log$ so that $-\pi / 3<\operatorname{Im} \varphi(u)<0$.

Remark 3.3. Note that $\varphi(u)$ and $\tilde{\varphi}(u)$ are purely imaginary since $\left|e^{u}+e^{-u}-1-\sqrt{\left(e^{u}+e^{-u}+1\right)\left(e^{u}+e^{-u}-3\right)}\right|=4$.

It is easy to see that $d \Phi\left(w_{0}\right) / d w=0$. Since we have

$$
\operatorname{Im}\left(w_{0}\right)+\frac{2 \pi}{u} \operatorname{Re}\left(w_{0}\right)=\frac{\operatorname{Im} \tilde{\varphi}(u)}{u},
$$

$w_{0}$ is in the domain of $\Phi$.

We choose a path $P$ from $\varepsilon$ to $1-\varepsilon$ that passes through $w_{0}$ so that near $w_{0}$ it keeps $\operatorname{Im} \Phi(w)$ constant and that $\operatorname{Re} \Phi(w)$ takes its maximum (over all $w$ on $P$ ) at $w_{0}$ as indicated in the thick curve in Figures 2. Then the integral $\int_{C_{-}(\varepsilon)} \exp (N \Phi(w)) d w$ is approximated by the integral near $w_{0}$ along the path we choose. More precisely we have

$$
\int_{C_{-}(\varepsilon)} \exp (N \Phi(w)) d w \underset{N \rightarrow \infty}{\sim} \frac{\sqrt{2 \pi} \exp \left(N \Phi\left(w_{0}\right)\right)}{\sqrt{N} \sqrt{-d^{2} \Phi\left(w_{0}\right) / d w^{2}}}
$$

from [16, Theorem 7.2.8], where the sign of the square root of $-d^{2} \Phi\left(w_{0}\right) / d w^{2}$ is chosen so that

$$
\sqrt{-d^{2} \Phi\left(w_{0}\right) / d w^{2}} \times\left(\text { tangent of } P \text { at } w_{0}\right)>0
$$

Note that

$$
\Phi\left(w_{0}\right)=\frac{1}{\xi}\left(\operatorname{Li}_{2}\left(e^{u-\varphi(u)}\right)-\operatorname{Li}_{2}\left(e^{u+\varphi(u)}\right)-u \tilde{\varphi}(u)\right) .
$$

From Propositions 3.1 and 3.2 , choosing $P$ as a contour in Proposition 3.2 we have

$$
\begin{aligned}
& \left|G_{ \pm}(N, \varepsilon) \mp \sqrt{-1} \int_{C_{ \pm}(\varepsilon)} \exp (N \Phi(w)) d w\right| \\
\leq & \left|\int_{C_{ \pm}(\varepsilon)}(\tan (N \pi w) \mp \sqrt{-1}) g_{N}(w) d w \pm \sqrt{-1} \int_{C_{ \pm}(\varepsilon)}\left(g_{N}(w)-\exp (N \Phi(w))\right) d w\right| \\
\leq & \frac{K_{1, \pm}}{N}+\frac{K_{2} \log (N)}{N} \exp \left(N \operatorname{Re} \Phi\left(w_{0}\right)\right) .
\end{aligned}
$$




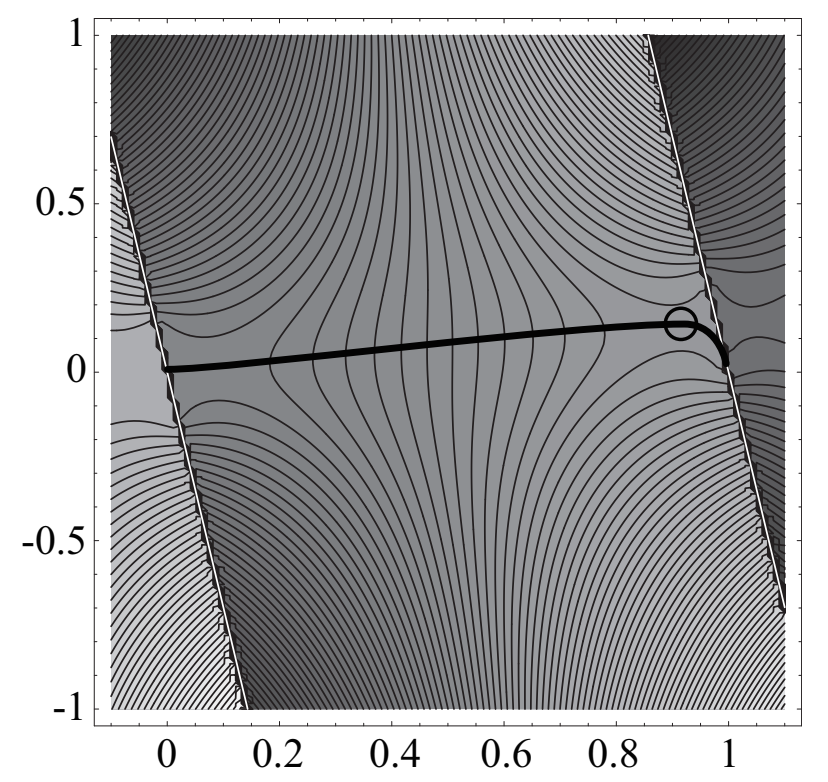

FiguRe 2. A contour plot of $\operatorname{Re} \Phi(w)$ on the complex plane for $u=0.9$. A brighter part is higher than a darker part. The path $P$ is indicated by a thick curve and the saddle point $w_{0}$ is marked by a circle.

From (3.2) we have

$$
\begin{aligned}
& \lim _{N \rightarrow \infty}\left|\frac{G_{ \pm}(N, \varepsilon)}{ \pm \sqrt{-1} \int_{C_{ \pm}(\varepsilon)} \exp (N \Phi(w)) d w}-1\right| \\
& \leq \frac{K_{1, \pm}}{N\left|\int_{C_{ \pm}(\varepsilon)} \exp (N \Phi(w)) d w\right|}+\frac{K_{2} \log (N)}{N} \times \frac{\exp \left(N \operatorname{Re} \Phi\left(w_{0}\right)\right)}{\left|\int_{C_{ \pm}(\varepsilon)} \exp (N \Phi(w)) d w\right|} \\
& \underset{N \rightarrow \infty}{\longrightarrow} 0
\end{aligned}
$$

Here we use the following lemma which will be proved in Section 3.4.

Lemma 3.4. The real part of $\Phi\left(w_{0}\right)$ is positive for $0<u<\log ((3+\sqrt{5}) / 2)$. Therefore from (3.2) we see that $\int_{C_{ \pm}(\varepsilon)} \exp (N \Phi(w)) d w$ grows exponentially.

So we have

$$
G_{ \pm}(N, \varepsilon) \underset{N \rightarrow \infty}{\sim} \pm \sqrt{-1} \int_{C_{ \pm}(\varepsilon)} \exp (N \Phi(w)) d w .
$$

Therefore from (2.4) we have

$$
\begin{aligned}
& J_{N}(K ; \exp (\xi / N)) \\
& \underset{N \rightarrow \infty}{\sim} \frac{e^{2 \pi \sqrt{-1} u N / \xi}}{e^{u}-1} \frac{N e^{u / 2}}{2}\left(\int_{C_{-}(\varepsilon)} \exp (N \Phi(w)) d w-\int_{C_{+}(\varepsilon)} \exp (N \Phi(w)) d w\right) \\
&=\frac{N e^{2 \pi \sqrt{-1} u N / \xi}}{2 \sinh (u / 2)}\left(\int_{C_{-}(\varepsilon)} \exp (N \Phi(w)) d w\right)
\end{aligned}
$$

from the lemma below. 
Lemma 3.5. For $\gamma=(2 \pi-\sqrt{-1} u) /(2 N)$ with positive $u$, we have

$$
\frac{S_{\gamma}(-\pi-\sqrt{-1} u+\gamma)}{S_{\gamma}(\pi-\sqrt{-1} u-\gamma)}=\frac{e^{\pi u / \gamma}-1}{e^{u}-1} .
$$

Therefore we have the following asymptotic equivalence:

$$
\frac{S_{\gamma}(-\pi-\sqrt{-1} u+\gamma)}{S_{\gamma}(\pi-\sqrt{-1} u-\gamma)} \underset{N \rightarrow \infty}{\sim} \frac{e^{2 \pi \sqrt{-1} u N / \xi}}{e^{u}-1}
$$

A proof of the lemma is given in Section 5 .

Remark 3.6. When $u=0$, we have

$$
\frac{S_{\gamma}(-\pi+\gamma)}{S_{\gamma}(\pi-\gamma)}=N
$$

from [1, P. 492].

Since

$$
\frac{d^{2} \Phi\left(w_{0}\right)}{d w^{2}}=\xi \sqrt{\left(e^{u}+e^{-u}+1\right)\left(e^{u}+e^{-u}-3\right)},
$$

we have the following asymptotic equivalence from (3.2):

$$
\int_{C_{-}(\varepsilon)} \exp (N \Phi(w)) d w \underset{N \rightarrow \infty}{\sim} \frac{\sqrt{2 \pi} \exp \left(N \Phi\left(w_{0}\right)\right)}{\sqrt{N} \sqrt{-\xi \sqrt{\left(e^{u}+e^{-u}+1\right)\left(e^{u}+e^{-u}-3\right)}}},
$$

where we choose the square root of $\sqrt{-\xi \sqrt{\left(e^{u}+e^{-u}+1\right)\left(e^{u}+e^{-u}-3\right)}}$ so that it is in the fourth quadrant from (3.3).

Therefore we finally have

$$
\begin{aligned}
& J_{N}(E ; \exp (\xi / N)) \\
\sim & \frac{N e^{2 \pi \sqrt{-1} u N / \xi}}{2 \sinh (u / 2)} \frac{\sqrt{2 \pi}}{\sqrt{N} \sqrt{-\xi \sqrt{\left(e^{u}+e^{-u}+1\right)\left(e^{u}+e^{-u}-3\right)}}} \\
& \times \exp \left(\frac{N}{\xi}\left(\operatorname{Li}_{2}\left(e^{u-\varphi(u)}\right)-\operatorname{Li}_{2}\left(e^{u+\varphi(u)}\right)-u \tilde{\varphi}(u)\right)\right) \\
= & \frac{\sqrt{\pi}}{2 \sinh (u / 2)} \sqrt{\frac{-2}{\sqrt{\left(e^{u}+e^{-u}+1\right)\left(e^{u}+e^{-u}-3\right)}}} \sqrt{\frac{N}{\xi}} \exp \left(\frac{N}{\xi} S(u)\right) .
\end{aligned}
$$

Here we put

$$
S(u):=\operatorname{Li}_{2}\left(e^{u-\varphi(u)}\right)-\operatorname{Li}_{2}\left(e^{u+\varphi(u)}\right)-u \varphi(u) .
$$

Remark 3.7. When $u=0$, we have

$$
\int_{C_{-}(\varepsilon)} \exp (N \Phi(w)) d w \underset{N \rightarrow \infty}{\sim} \frac{\exp \left(N \Phi\left(w_{0}\right)\right)}{\sqrt{N} 3^{1 / 4}} .
$$

Since $w_{0}=5 / 6$ in this case we have

$$
\begin{aligned}
\Phi\left(w_{0}\right) & =\frac{1}{2 \pi \sqrt{-1}}\left(\operatorname{Li}_{2}\left(e^{-5 \pi \sqrt{-1} / 3}\right)-\operatorname{Li}_{2}\left(e^{5 \pi \sqrt{-1} / 3}\right)\right) \\
& =\frac{6 \sqrt{-1} \Lambda(\pi / 3)}{2 \pi \sqrt{-1}}=\frac{\operatorname{Vol}(E)}{2 \pi} .
\end{aligned}
$$

Therefore from Remark 3.6, we have Theorem 1.3 ,

4. Topological interpretations of $S(u)$ And $T(u)$

In this section we describe topological interpretations of $S(u)$ and $T(u)$. 
4.1. Representation. Let $x$ and $y$ be the Wirtinger generators of the fundamental group $\pi_{1}\left(S^{3} \backslash E\right)$ (with a base point above the paper) of the complement of the figure-eight knot $E$ depicted in Figure 3. The group $\pi_{1}\left(S^{3} \backslash E\right)$ has the following

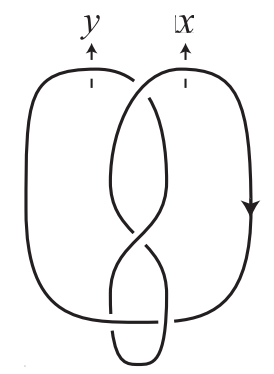

FiguRE 3. Generators of the fundamental group of the complement of the figure-eight knot

presentation:

$$
\pi_{1}\left(S^{3} \backslash E\right)=\left\langle x, y \mid x y^{-1} x^{-1} y x=y x y^{-1} x^{-1} y\right\rangle .
$$

Due to [25] any non-abelian representation $\rho$ of $\pi_{1}\left(S^{3} \backslash E\right)$ into $S L(2 ; \mathbb{C})$ is, up to conjugation, given as follows:

$$
\begin{aligned}
& \rho(x):=\left(\begin{array}{cc}
m^{1 / 2} & 1 \\
0 & m^{-1 / 2}
\end{array}\right), \\
& \rho(y):=\left(\begin{array}{cc}
m^{1 / 2} & 0 \\
-d & m^{-1 / 2}
\end{array}\right),
\end{aligned}
$$

where

$$
d=\frac{1}{2}\left(m+m^{-1}-3 \pm \sqrt{\left(m+m^{-1}+1\right)\left(m+m^{-1}-3\right)}\right) .
$$

Since the longitude $\lambda$ is given by $x y^{-1} x y x^{-2} y x y^{-1} x^{-1}$ if we read it off from the top right, we have

$$
\rho(\lambda)=\left(\begin{array}{cc}
\ell(m)^{ \pm 1} & \left(m^{1 / 2}+m^{-1 / 2}\right) \sqrt{\left(m+m^{-1}+1\right)\left(m+m^{-1}-3\right)} \\
0 & \ell(m)^{\mp 1}
\end{array}\right),
$$

where

$\ell(m):=\frac{m^{2}-m-2-m^{-1}+m^{-2}}{2}+\frac{\left(m-m^{-1}\right)}{2} \sqrt{\left(m+m^{-1}+1\right)\left(m+m^{-1}-3\right)}$.

See also [19, Section 3.1].

Let $\rho_{u}$ be the representation given by putting $m:=e^{u}$. We introduce a parameter $v$ so that $\ell\left(e^{u}\right)=e^{v / 2}$. Since we assume that $0<u<\log ((3+\sqrt{5}) / 2)$, we have $2<e^{u}+e^{-u}<3$. Therefore we have

$\left|\ell\left(e^{u}\right)\right|^{2}=\frac{1}{4}\left(e^{2 u}-e^{u}-2-e^{-u}+e^{-2 u}\right)^{2}-\frac{1}{4}\left(e^{u}-e^{-u}\right)^{2}\left(e^{u}+e^{-u}+1\right)\left(e^{u}+e^{-u}-3\right)=1$ and so $v$ is purely imaginary.

The representation $\rho_{u}$ gives an incomplete hyperbolic structure to $S^{3} \backslash E$ and its completion is the generalized Dehn surgery [26] with parameter $(p, q)$ with $p u+q v=$ $2 \pi \sqrt{-1}$. Since $v$ is purely imaginary $p=0$ and $q=2 \pi \sqrt{-1} / v$. Therefore the completion is a cone manifold whose underlying space is the 0 -surgery of $S^{3}$ along the figure-eight knot with singularity the core of the surgery and with cone angle $\alpha=\operatorname{Im}(v)=v / \sqrt{-1}$. Note that when $u=0$, the cone angle is $2 \pi$ and when $u=\log ((3+\sqrt{5}) / 2)$, the cone angle is 0 . See [10] for more details about the geometric structure of this manifold. 
In the following two subsections we will calculate the Reidemeister torsion and the Chern-Simons invariant associated with $\rho_{u}$.

4.2. Reidemeister torsion. From [24, P. 113] (see also [4, $\S$ 6.3]) the cohomological Reidemeister torsion $T_{\lambda}^{E}\left(\rho_{u}\right)$ associated with the longitude $\lambda$ twisted by the adjoint action of the representation $\rho_{u}$ is given by

$$
T_{\lambda}^{E}\left(\rho_{u}\right)=\frac{1}{\sqrt{17+4 \operatorname{Tr}\left(\rho_{u}(\lambda)\right)}}=\frac{1}{2 m+2 m^{-1}-1}
$$

up to sign, where Tr means the trace. Note that since in 24] Porti uses homological Reidemeister torsion, we need to take the inverse.

From [24, Théorème 4.1] the Reidemeister torsion $T_{\mu}^{E}\left(\rho_{u}\right)$ associated with the meridian $\mu$ is given by

$$
T_{\mu}^{E}\left(\rho_{u}\right)= \pm \frac{\partial v}{\partial u} T_{\lambda}^{E}\left(\rho_{u}\right)
$$

Since $\ell\left(e^{u}\right)=e^{v / 2}$, we have

$$
\pm T_{\mu}^{E}\left(\rho_{u}\right)=\frac{\partial\left(2 \log \ell\left(e^{u}\right)\right)}{\partial u} \times \frac{1}{2 e^{u}+2 e^{-u}-1}=\frac{2}{\sqrt{\left(e^{u}+e^{-u}+1\right)\left(e^{u}+e^{-u}-3\right)}} .
$$

Therefore $T(u)$ that appears in Theorem 1.4 coincides with $T_{\mu}^{E}\left(\rho_{u}\right)$ up to sign.

4.3. Chern-Simons invariant. Let $M$ be a closed three-manifold and $\rho: \pi_{1}(M) \rightarrow \mathrm{SL}(2 ; \mathbb{C})$ a representation. Then the Chern-Simons invariant $\operatorname{cs}_{M}(\rho)$ is defined as

$$
\frac{1}{8 \pi^{2}} \int_{M} \operatorname{Tr}\left(A \wedge d A+\frac{2}{3} A \wedge A \wedge A\right) \in \mathbb{C} / \mathbb{Z},
$$

where $A$ is the $\mathfrak{s l}(2 ; \mathbb{C})$-valued 1 -form on $M$ with $d A+A \wedge A=0$ such that $\rho$ is given as the holonomy representation induced by the flat connection on $M \times \operatorname{SL}(2 ; \mathbb{C})$ defined by $A$.

In [14] Kirk and Klassen defined the $\operatorname{SL}(2 ; \mathbb{C})$ Chern-Simons invariant $\operatorname{cs}_{M}(\rho)$ for a three-manifold with boundary. It is a triple $[\alpha, \beta ; z]$ of complex numbers modulo the following relation:

$$
[\alpha, \beta ; z]=[\alpha+1, \beta ; z \exp (-8 \pi \sqrt{-1} \beta)]=[\alpha, \beta+1 ; z \exp (8 \pi \sqrt{-1} \alpha)]=[-\alpha,-\beta ; z] .
$$

For $i=1,2$, let $M_{i}$ be a three-manifold with boundary $\partial M_{i}$ a torus and put $M:=$ $M_{1} \bigcup_{\partial M_{1}=-\partial M_{2}} M_{2}$. For a representation $\rho: \pi_{1}(M) \rightarrow S L(2 ; \mathbb{C})$, put $\rho_{i}:=\left.\rho\right|_{M_{i}}$. If $\operatorname{cs}_{M_{i}}\left(\rho_{i}\right)=\left[\alpha, \beta ; z_{i}\right]$ then $\operatorname{cs}_{M}(\rho)$ is given by $z_{1} z_{2}$.

Define the Chern-Simons invariant $\operatorname{CS}_{u}(K)$ for a knot $K$ to be

$$
\operatorname{cs}_{S^{3} \backslash E}\left(\rho_{u}\right)=\left[\frac{u}{4 \pi \sqrt{-1}}, \frac{v}{4 \pi \sqrt{-1}} ; \exp \left(\frac{2}{\pi \sqrt{-1}} \operatorname{CS}_{u}(K)\right)\right] .
$$

Then as described in 9] we have

$$
\mathrm{CS}_{u}(E)=S(u)-\pi \sqrt{-1} u-\frac{u v}{4} .
$$

Note that we are using the $\operatorname{PSL}(2 ; \mathbb{C})$ normalization of the Chern-Simons invariant 14. P. 543]. So the function $f(u)$ in $\left[23\right.$ is $-\mathrm{CS}_{u}(E) / 4$ (up to a constant) and the function $f(u)$ in [14, P. 543] and [27] is $-2 \sqrt{-1} \mathrm{CS}_{u}(E) / \pi$. 


\section{Calculation of $S_{\gamma}$}

In this section we first show the analyticity of $S_{\gamma}$ and then calculate its special values.

Lemma 5.1. If a complex number $\gamma$ has the positive real part, then $S_{\gamma}(z)$ is an analytic function in $\{z \in \mathbb{C}|| \operatorname{Re}(z) \mid<\pi+\operatorname{Re}(\gamma)\}$.

Proof. Put

$$
L_{\gamma}(t):=\frac{e^{z t}}{t \sinh (\pi t) \sinh (\gamma t)}
$$

We will show that the improper integrals $\int_{R}^{\infty} L_{\gamma} t d t$ and $\int_{-\infty}^{R} L_{\gamma} t d t$ converge.

Putting $z:=x+\sqrt{-1} y$ and $\gamma:=a+\sqrt{-1} b$ for real numbers $x, y, a, b$ with $a>0$ we have

$$
\operatorname{Re}\left(L_{\gamma}(t)\right)=\frac{-2 e^{x t}(\cosh (a t) \sin (b t) \sin (t y)+\sinh (a t) \cos (b t) \cos (y t))}{t \sinh (\pi t)(\cos (2 b t)-\cosh (2 a t))}
$$

and

$$
\operatorname{Im}\left(L_{\gamma}(t)\right)=\frac{2 e^{x t}(\cosh (a t) \sin (b t) \cos (t y)-\sinh (a t) \cos (b t) \sin (y t))}{t \sinh (\pi t)(\cos (2 b t)-\cosh (2 a t))}
$$

for $t \in \mathbb{R}$.

If $t$ is positive and sufficiently large we have

$$
\begin{aligned}
\left|\operatorname{Re}\left(L_{\gamma}(t)\right)\right| & \leq \frac{2 e^{x t} e^{a t}}{t \sinh (\pi t)(\cosh (2 a t)-1)} \\
& =\frac{8 e^{(x-\pi-a) t}}{t\left(1-e^{-2 \pi t}\right)\left(1+e^{-4 a t}-2 e^{-2 a t}\right)}
\end{aligned}
$$

and similarly we have

$$
\left|\operatorname{Im}\left(L_{\gamma}(t)\right)\right| \leq \frac{8 e^{(x-\pi-a) t}}{t\left(1-e^{-2 \pi t}\right)\left(1+e^{-4 a t}-2 e^{-2 a t}\right)}
$$

Therefore the integral $\int_{R}^{\infty} L_{\gamma} t d t$ converges since $x<\pi+a$.

If $t$ is negative and $|t|$ is sufficiently large we have

$$
\begin{aligned}
\left|\operatorname{Re}\left(L_{\gamma}(t)\right)\right| & \leq \frac{2 e^{(x-a) t}}{t \sinh (\pi t)(\cosh (2 a t)-1)} \\
& =\frac{8 e^{(x+\pi+a) t}}{t\left(e^{2 \pi t}-1\right)\left(e^{4 a t}+1-2 e^{2 a t}\right)}
\end{aligned}
$$

and similarly we have

$$
\left|\operatorname{Im}\left(L_{\gamma}(t)\right)\right| \leq \frac{8 e^{(x+\pi+a) t}}{t\left(e^{2 \pi t}-1\right)\left(e^{4 a t}+1-2 e^{2 a t}\right)}
$$

Therefore the integral $\int_{-\infty}^{R} L_{\gamma} t d t$ converges since $x>-\pi-a$.

Next we prove Lemma 3.5 
Proof of Lemma 3.5. By the definition we have

$$
\begin{aligned}
& \frac{S_{\gamma}(-\pi-\sqrt{-1} u+\gamma)}{S_{\gamma}(\pi-\sqrt{-1} u-\gamma)} \\
= & \exp \left(\frac{1}{4} \int_{C_{R}}\left(\frac{e^{(-\pi-\sqrt{-1} u+\gamma) t}}{\sinh (\pi t) \sinh (\gamma t)}-\frac{e^{(\pi-\sqrt{-1} u-\gamma) t}}{\sinh (\pi t) \sinh (\gamma t)}\right) \frac{d t}{t}\right) \\
= & \exp \left(\frac{1}{2} \int_{C_{R}} e^{-\sqrt{-1} u t} \frac{\sinh (-\pi t+\gamma t)}{\sinh (\pi t) \sinh (\gamma t)} \frac{d t}{t}\right) \\
= & \exp \left(\frac{1}{2} \int_{C_{R}} \frac{e^{-\sqrt{-1} u t} \operatorname{coth}(\pi t)}{t} d t-\frac{1}{2} \int_{C_{R}} \frac{e^{-\sqrt{-1} u t} \operatorname{coth}(\gamma t)}{t} d t\right) .
\end{aligned}
$$

We will calculate the integral $\int_{C_{R}} e^{-\sqrt{-1} u t} \operatorname{coth}(\kappa t) / t d t$ for a complex number $\kappa$ with $\operatorname{Re}(\kappa)>0$ and $\operatorname{Im}(\kappa) \leq 0$.

Put $\kappa:=\alpha-\beta \sqrt{-1}$ with $\alpha>0$ and $\beta \geq 0$. For a positive number $r$, let $U_{1}$ be the segment connecting $r$ and $r+r^{\prime} \beta / \alpha-r^{\prime} \sqrt{-1}, U_{2}$ be the segment connecting $r+r^{\prime} \beta / \alpha-r^{\prime} \sqrt{-1}$ and $-r+r^{\prime} \beta / \alpha-r^{\prime} \sqrt{-1}$, and $U_{3}$ be the segment connecting $-r+r^{\prime} \beta / \alpha-r^{\prime} \sqrt{-1}$ and $-r$ with $r^{\prime}:=(n+1 / 2) \pi \alpha /|\kappa|^{2}$, where $n:=\left\lfloor r|\kappa|^{2} /(\pi \alpha)\right\rfloor$. Here $\lfloor x\rfloor$ is the largest integer that does not exceed $x$. Note that $r-\pi \alpha /\left(2|\kappa|^{2}\right)<$ $r^{\prime} \leq r+\pi \alpha /\left(2|\kappa|^{2}\right)$. We use $r^{\prime}$ instead of $r$ just to avoid the poles of $\operatorname{coth}(\kappa t)$. Then we have

$$
\begin{aligned}
& \left|\int_{U_{1}} \frac{e^{-\sqrt{-1} u t}}{t} \operatorname{coth}(\kappa t) d t\right| \\
\leq & \int_{0}^{r^{\prime}}\left|\frac{e^{-\sqrt{-1} u(r+s \beta / \alpha-s \sqrt{-1})}}{r+s \beta / \alpha-s \sqrt{-1}}\right||\operatorname{coth}((\alpha-\beta \sqrt{-1})(r+s \beta / \alpha-s \sqrt{-1}))| d s \\
\leq & \frac{1}{r} \int_{0}^{r^{\prime}} e^{-u s}\left|\operatorname{coth}\left(r \alpha-\sqrt{-1}\left(s \alpha+r \beta+s \beta^{2} / \alpha\right)\right)\right| d s \\
\leq & \frac{1}{r} \int_{0}^{r^{\prime}} e^{-u s} d s \\
= & \frac{1}{u r}\left(1-e^{-u r^{\prime}}\right) \stackrel{r \rightarrow \infty}{\longrightarrow} 0 .
\end{aligned}
$$

Similarly we have

$$
\begin{aligned}
& \left|\int_{U_{3}} \frac{e^{-\sqrt{-1} u t}}{t} \operatorname{coth}(\kappa t) d t\right| \\
\leq & \int_{0}^{r^{\prime}}\left|\frac{e^{-\sqrt{-1} u(-r+s \beta / \alpha-s \sqrt{-1})}}{-r+s \beta / \alpha-s \sqrt{-1}}\right||\cosh ((\alpha-\beta \sqrt{-1})(-r+s \beta / \alpha-s \sqrt{-1}))| d s \\
\leq & \frac{|\kappa|}{r \alpha} \int_{0}^{r^{\prime}} e^{-u s}\left|\cosh \left(-r \alpha-\sqrt{-1}\left(s \alpha-r \beta+s \beta^{2} / \alpha\right)\right)\right| d s \\
\leq & \frac{|\kappa|}{u r \alpha}\left(1-e^{-u r^{\prime}}\right) \stackrel{r \rightarrow \infty}{\longrightarrow} 0 .
\end{aligned}
$$


We also have

$$
\begin{aligned}
& \left|\int_{U_{2}} \frac{e^{-\sqrt{-1} u t}}{t} \operatorname{coth}(\kappa t) d t\right| \\
& \leq \int_{-r}^{r}\left|\frac{e^{-\sqrt{-1} u\left(s+r^{\prime} \beta / \alpha-r^{\prime} \sqrt{-1}\right)}}{s+r^{\prime} \beta / \alpha-r^{\prime} \sqrt{-1}}\right|\left|\operatorname{coth}\left((\alpha-\beta \sqrt{-1})\left(s+r^{\prime} \beta / \alpha-r^{\prime} \sqrt{-1}\right)\right)\right| d s \\
& \leq \frac{e^{-u r^{\prime}}}{r^{\prime}} \int_{-r}^{r}\left|\operatorname{coth}\left(s \alpha-\sqrt{-1}\left(r^{\prime} \alpha+s \beta+r^{\prime} \beta^{2} / \alpha\right)\right)\right| d s \\
& =\frac{e^{-u r^{\prime}}}{r^{\prime}} \int_{-r}^{r}|\operatorname{coth}(s \alpha-\sqrt{-1}((n+1 / 2) \pi+s \beta))| d s \\
& =\frac{e^{-u r^{\prime}}}{r^{\prime}} \int_{-r}^{r}|\tanh (\kappa s)| d s \\
& \left(\delta:=\max _{-1 \leq s \leq 1}|\tanh (\kappa s)|>0\right) \\
& \leq \frac{e^{-u r^{\prime}}}{r^{\prime}}\left(2 \delta+\int_{-r}^{-1} \frac{\left|e^{\kappa s}-e^{-\kappa s}\right|}{\left|e^{\kappa s}+e^{-\kappa s}\right|} d s+\int_{1}^{r} \frac{\left|e^{\kappa s}-e^{-\kappa s}\right|}{\left|e^{\kappa s}+e^{-\kappa s}\right|} d s\right) \\
& \leq \frac{e^{-u r^{\prime}}}{r^{\prime}}\left(2 \delta+\int_{-r}^{-1} \frac{\left|e^{\kappa s}\right|+\left|e^{-\kappa s}\right|}{|| e^{\kappa s}|-| e^{-\kappa s}||} d s+\int_{1}^{r} \frac{\left|e^{\kappa s}\right|+\left|e^{-\kappa s}\right|}{|| e^{\kappa s}|-| e^{-\kappa s}||} d s\right) \\
& =\frac{2 e^{-u r^{\prime}}}{r^{\prime}}\left(\delta+\int_{1}^{r}|\operatorname{coth}(\alpha s)| d s\right) \\
& =\frac{2 e^{-u r^{\prime}}}{r^{\prime}}\left(\delta+\frac{\log (\sinh (\alpha r))-\log (\sinh (\alpha))}{\alpha}\right) \stackrel{r \rightarrow \infty}{\longrightarrow} 0 .
\end{aligned}
$$

Therefore we have

$$
\begin{aligned}
\int_{C_{R}} \frac{e^{-\sqrt{-1} u t}}{t} \operatorname{coth}(\kappa t) d t & =2 \pi \sqrt{-1} \sum_{l=1}^{\infty} \operatorname{Res}\left(\frac{e^{-\sqrt{-1} u t}}{t} \operatorname{coth}(\kappa t) ; t=\frac{l \pi \sqrt{-1}}{\kappa}\right) \\
& =2 \pi \sqrt{-1} \sum_{l=1}^{\infty} \frac{e^{l u \pi / \kappa}}{l \pi \sqrt{-1}} \\
& =-2 \log \left(1-e^{u \pi / \kappa}\right)
\end{aligned}
$$

and so we have

$$
\frac{S_{\gamma}(-\pi-\sqrt{-1} u+\gamma)}{S_{\gamma}(\pi-\sqrt{-1} u-\gamma)}=\frac{1-e^{u \pi / \gamma}}{1-e^{u}}
$$

\section{Proof of Proposition 3.1}

In this section we follow [1, Appendix A] to show Proposition 3.1.

From [1, $\S 4.1$ ] we have the following integral expression for $|\operatorname{Re}(z)|<\pi$, or $|\operatorname{Re}(z)|=\pi$ and $\operatorname{Im}(z) \geq 0$ :

$$
\frac{1}{2 \sqrt{-1}} \operatorname{Li}_{2}\left(-e^{\sqrt{-1} z}\right)=\frac{1}{4} \int_{C_{R}} \frac{e^{z t}}{t^{2} \sinh (\pi t)} d t .
$$

Therefore we have

$$
\begin{aligned}
S_{\gamma}(z) & =\exp \left(\frac{1}{2 \sqrt{-1} \gamma} \operatorname{Li}_{2}\left(-e^{\sqrt{-1} z}\right)+I_{\gamma}(z)\right) \\
& =\exp \left(\frac{N}{\xi} \operatorname{Li}_{2}\left(-e^{\sqrt{-1} z}\right)+I_{\gamma}(z)\right)
\end{aligned}
$$


where

$$
I_{\gamma}(z):=\frac{1}{4} \int_{C_{R}} \frac{e^{z t}}{t \sinh (\pi t)}\left(\frac{1}{\sinh (\gamma t)}-\frac{1}{\gamma t}\right) d t
$$

(see [1, Equation (4.2)]). Note that $I_{\gamma}(z)$ is defined for $z$ with $|\operatorname{Re}(z)| \leq \pi([1$, Appendix A]).

Then we have from (2.3)

$$
\begin{aligned}
& g_{N}(z) \\
= & \exp \left(\frac{N}{\xi}\left(\operatorname{Li}_{2}\left(-e^{\sqrt{-1} \pi+u-\xi z}\right)-\operatorname{Li}_{2}\left(-e^{-\sqrt{-1} \pi+u+\xi z}\right)\right)-N u z\right) \\
& \times \exp \left(I_{\gamma}(\pi-\sqrt{-1} u+\sqrt{-1} \xi z)-I_{\gamma}(-\pi-\sqrt{-1} u-\sqrt{-1} \xi z)\right) \\
= & \exp (N \Phi(z)) \exp \left(I_{\gamma}(\pi-\sqrt{-1} u+\sqrt{-1} \xi z)-I_{\gamma}(-\pi-\sqrt{-1} u-\sqrt{-1} \xi z)\right) .
\end{aligned}
$$

We first give an estimation for $\left|I_{\gamma}(z)\right|$.

Lemma 6.1 (see Lemma 3 in [1]). If $|\operatorname{Re}(z)|<\pi$, then we have

$$
\left|I_{\gamma}(z)\right| \leq A\left(\frac{1}{\pi-\operatorname{Re}(z)}+\frac{1}{\pi+\operatorname{Re}(z)}\right)|\gamma|+B\left(1+e^{-\operatorname{Im}(z) R}\right)|\gamma|
$$

If $|\operatorname{Re}(z)| \leq \pi$, then we have

$$
\left|I_{\gamma}(z)\right| \leq 2 A+B\left(1+e^{-\operatorname{Im}(z) R}\right)|\gamma|
$$

Proof. We follow [1, Appendix A].

Put

$$
\psi(w):=\frac{1}{\sinh (w)}-\frac{1}{w},
$$

which is holomorphic in the open disk $D_{0}(\pi)$ with center 0 and radius $\pi$. Note that

$$
\psi(w)=\frac{w-\sinh (w)}{w \sinh (w)}=-w \frac{h(w)}{k(w)}
$$

for entire functions $h(w)$ and $k(w)$ with

$$
h(w)=\sum_{j=0}^{\infty} \frac{w^{2 j}}{(2 j+3) !}
$$

and

$$
k(w)=\sum_{j=0}^{\infty} \frac{w^{2 j}}{(2 j+1) !}
$$

when $|w|<\pi$. Therefore there exists $\delta>0$ such that $\min _{|w| \leq \delta}|\psi(w) / w|=D>0$ since $\lim _{w \rightarrow 0} \psi(w) / w=1 / 6$.

Put $C_{\delta}:=[-\delta /|\gamma|,-R] \cup \Omega_{R} \cup[R, \delta /|\gamma|]$. Consider the following integrals $I_{0}(z)$ and $I_{1}(z)$ so that $I_{\gamma}(z)=I_{0}(z)+I_{1}(z)$ :

$$
\begin{aligned}
& I_{0}(z):=\frac{1}{4} \int_{C_{\delta}} \frac{\exp (z t)}{t \sinh (\pi t)} \psi(\gamma t) d t \\
& I_{1}(z):=\frac{1}{4} \int_{-\infty}^{-\delta /|\gamma|} \frac{\exp (z t)}{t \sinh (\pi t)} \psi(\gamma t) d t+\frac{1}{4} \int_{\delta /|\gamma|}^{\infty} \frac{\exp (z t)}{t \sinh (\pi t)} \psi(\gamma t) d t .
\end{aligned}
$$

Since $\lim _{w \rightarrow \infty} w \psi(w)=0, \psi(w)$ has poles at $w=m \pi \sqrt{-1}(m= \pm 1, \pm 2, \ldots)$, and $\operatorname{Im}(\gamma) \neq 0$, we have $|\gamma t \psi(\gamma t)| \leq E$ for a positive number $E$. Note that $E$ depends only on the argument of $\gamma$ and so only on $\xi=\gamma \times 2 \sqrt{-1} N$. So we have

$$
|\psi(\gamma t)| \leq \frac{E}{|\gamma t|}
$$


for any $t$. Therefore we can apply the argument (replacing $\gamma$ there with $|\gamma| / E$ and $a$ with $\delta /|\gamma|)$ in [1, Page 532] to conclude

$$
\left|I_{1}(z)\right| \leq \frac{E}{\delta\left(1-e^{-2 \pi|\delta| /|\gamma|}\right)}
$$

if $|\operatorname{Re}(z)| \leq \pi$ and

$$
\left|I_{1}(z)\right| \leq \frac{E|\gamma|}{2 \delta^{2}\left(1-e^{-2 \pi \delta /|\gamma|)}\right.}\left(\frac{e^{-(\pi-\operatorname{Re}(z)) \delta /|\gamma|)}}{\pi-\operatorname{Re}(z)}+\frac{e^{-(\pi+\operatorname{Re}(z)) \delta /|\gamma|)}}{\pi+\operatorname{Re}(z)}\right)
$$

if $|\operatorname{Re}(z)|<\pi$.

Next we estimate $I_{0}(z)$. Let $M(z, R)$ be the maximum of $\left|\frac{e^{z t}}{t \sinh (\pi t)} \psi(\gamma t)\right|$ for $t \in \Omega_{R}$. Then we have

$$
\left|\int_{\Omega_{R}} \frac{\exp (z t)}{t \sinh (\pi t)} \psi(\gamma t) d t\right| \leq \pi R M(z, R) .
$$

From (6.2) we have

$$
\begin{aligned}
M(z, R) & =\max _{t \in \Omega_{R}}\left|\frac{e^{z t}}{t \sinh (\pi t)} \psi(\gamma t)\right| \\
& =\max _{t \in \Omega_{R}}|\gamma|\left|\frac{e^{z t}}{\sinh (\pi t)}\right|\left|\frac{h(\gamma t)}{k(\gamma t)}\right|
\end{aligned}
$$

since $|\gamma t|=|\gamma| R<\pi$ when $t \in \Omega_{R}$ and $R<1$. Then putting $L(R):=$ $\max _{t \in \Omega_{R}}|h(z) / k(z)|$ and $N(z, R):=\max _{t \in \Omega_{R}}\left|e^{z t} /\left(e^{\pi t}-e^{-\pi t}\right)\right|$ we can apply the argument in [1, Page 533] to have the following estimation.

$$
\left|\frac{1}{4} \int_{\Omega_{R}} \frac{e^{z t}}{t \sinh (\pi t)} \psi(\gamma t) d t\right| \leq|\gamma| B\left(1+e^{-\operatorname{Im}(z) R}\right)
$$

for a constant $B$ (depending only on $R$ ).

Now we will estimate the rest of $I_{0}(z)$. We have

$$
\begin{aligned}
& \left|\int_{-\delta /|\gamma|}^{-R} \frac{e^{z t}}{t \sinh (\pi t)} \psi(\gamma t) d t+\int_{R}^{\delta /|\gamma|} \frac{e^{z t}}{t \sinh (\pi t)} \psi(\gamma t) d t\right| \\
\leq & \int_{-\delta / \mid \gamma}^{-R} \frac{e^{\operatorname{Re}(z) t}}{|t||\sinh (\pi t)|}|\psi(\gamma t)| d t+\int_{R}^{\delta / \mid \gamma} \frac{e^{\operatorname{Re}(z) t}}{|t||\sinh (\pi t)|}|\psi(\gamma t)| d t \\
= & \int_{R}^{\delta / \mid \gamma} \frac{e^{\operatorname{Re}(z) t}+e^{-\operatorname{Re}(z) t}}{t \sinh (\pi t)}|\psi(\gamma t)| d t \\
= & 2|\gamma| \int_{R}^{\delta / \mid \gamma} \frac{e^{\operatorname{Re}(z) t}+e^{-\operatorname{Re}(z) t}}{e^{\pi t}-e^{-\pi t}}\left|\frac{\psi(\gamma t)}{\gamma t}\right| d t .
\end{aligned}
$$

Since $\psi(w) / w$ is continuous in $D_{0}(\pi)$ and $\lim _{w \rightarrow 0} \psi(w) / w=1 / 6$, there exists $\delta>0$ such that $\min _{|w|<\delta}|\psi(w) / w|=D>0$. Therefore if $a<\delta /|\gamma|$, then $|\gamma t| \leq|\gamma| a<\delta$ in the integral and so we have

$$
\begin{aligned}
& \quad\left|\int_{-\delta /|\gamma|}^{-R} \frac{e^{z t}}{t \sinh (\pi t)} \psi(\gamma t) d t+\int_{R}^{\delta /|\gamma|} \frac{e^{z t}}{t \sinh (\pi t)} \psi(\gamma t) d t\right| \\
& \leq 2|\gamma| D \int_{R}^{\delta /|\gamma|} \frac{e^{\operatorname{Re}(z) t}+e^{-\operatorname{Re}(z) t}}{e^{\pi t}-e^{-\pi t}} d t \\
& \leq \frac{2|\gamma| D}{1-e^{-2 \pi R}} \int_{R}^{a}\left(e^{-(\pi-\operatorname{Re}(z)) t}+e^{-(\pi+\operatorname{Re}(z)) t}\right) d t .
\end{aligned}
$$


Then from the argument in [1, Page 533] we have

$$
\begin{aligned}
& \quad\left|\int_{-\delta /|\gamma|}^{-R} \frac{e^{z t}}{t \sinh (\pi t)} \psi(\gamma t) d t+\int_{R}^{\delta /|\gamma|} \frac{e^{z t}}{t \sinh (\pi t)} \psi(\gamma t) d t\right| \\
& \leq \frac{4 \delta D}{1-e^{-2 \pi R}}
\end{aligned}
$$

when $|\operatorname{Re}(z)| \leq \pi$, and

$$
\begin{aligned}
& \left|\int_{-\delta /|\gamma|}^{-R} \frac{e^{z t}}{t \sinh (\pi t)} \psi(\gamma t) d t+\int_{R}^{\delta /|\gamma|} \frac{e^{z t}}{t \sinh (\pi t)} \psi(\gamma t) d t\right| \\
\leq & \frac{2|\gamma| D}{\left(1-e^{-2 \pi R}\right)}\left(\frac{1-e^{-(\pi-\operatorname{Re}(z)) \delta /|\gamma|}}{\pi-\operatorname{Re}(z)}+\frac{1-e^{-(\pi+\operatorname{Re}(z)) \delta /|\gamma|}}{\pi+\operatorname{Re}(z)}\right)
\end{aligned}
$$

when $|\operatorname{Re}(z)|<\pi$.

Therefore if $|\operatorname{Re}(z)| \leq \pi$, we have

$$
\begin{aligned}
I_{\gamma}(z) & \leq \frac{E}{\delta\left(1-e^{-2 \pi|\delta| /|\gamma|}\right)}+|\gamma| B\left(1+e^{\operatorname{Im}(z) R}\right)+\frac{\delta D}{1-e^{-2 \pi R}} \\
& \leq \frac{1}{1-e^{-2 \pi R}}\left(\frac{E}{\delta}+\delta D\right)+|\gamma| B\left(1+e^{\operatorname{Im}(z) R}\right)
\end{aligned}
$$

since $\delta /|\gamma| \geq R$. If $|\operatorname{Re}(z)|<\pi$ we also have

$$
\begin{aligned}
& \left|I_{\gamma}(z)\right| \\
\leq & \frac{E|\gamma|}{2 \delta^{2}\left(1-e^{-2 \pi \delta /|\gamma|)}\right.}\left(\frac{e^{-(\pi-\operatorname{Re}(z)) \delta /|\gamma|)}}{\pi-\operatorname{Re}(z)}+\frac{e^{-(\pi+\operatorname{Re}(z)) \delta /|\gamma|)}}{\pi+\operatorname{Re}(z)}\right) \\
& +\frac{|\gamma| D}{2\left(1-e^{-2 \pi R}\right)}\left(\frac{1-e^{-(\pi-\operatorname{Re}(z)) \delta /|\gamma|}}{\pi-\operatorname{Re}(z)}+\frac{1-e^{-(\pi+\operatorname{Re}(z)) \delta /|\gamma|}}{\pi+\operatorname{Re}(z)}\right) \\
& +|\gamma| B\left(1+e^{\operatorname{Im}(z) R}\right) \\
\leq & |\gamma|\left(\frac{1}{\pi-\operatorname{Re}(z)}+\frac{1}{\pi+\operatorname{Re}(z)}\right) \frac{1}{1-e^{-2 \pi R}}\left(\frac{E}{2 \delta^{2}}+\frac{D}{2}\right)+|\gamma| B\left(1+e^{\operatorname{Im}(z) R}\right) .
\end{aligned}
$$

The lemma follows by putting

$$
A:=\frac{1}{1-e^{-2 \pi R}} \times \max \left\{\left(\frac{E}{2 \delta}+\frac{\delta D}{2}\right),\left(\frac{E}{2 \delta^{2}}+\frac{D}{2}\right)\right\} .
$$

Now we prove Proposition 3.1 .

First note that since $g_{N}(x)$ has no poles inside $C_{+}(\varepsilon) \cup C_{-}(\varepsilon)$, we can assume that $\varepsilon=0$ without changing the sum, that is,

$$
\begin{aligned}
& \int_{C_{+}(\varepsilon)}(\tan (N \pi x)-\sqrt{-1}) g_{N}(x) d x+\int_{C_{-}(\varepsilon)}(\tan (N \pi x)+\sqrt{-1}) g_{N}(x) d x \\
= & \int_{C_{+}(0)}(\tan (N \pi x)-\sqrt{-1}) g_{N}(x) d x+\int_{C_{-}(0)}(\tan (N \pi x)+\sqrt{-1}) g_{N}(x) d x
\end{aligned}
$$

We decompose $C_{+}(0)$ into $C_{+, 1} \cup C_{+, 2} \cup C_{+, 3}$, where $C_{+, 1}$ connects 0 and $-u /(2 \pi)+\sqrt{-1}, C_{+, 2}$ connects $-u /(2 \pi)+\sqrt{-1}$ and $1-u /(2 \pi)+\sqrt{-1}$, and $C_{+, 3}$ connects $1-u /(2 \pi)+\sqrt{-1}$ and 1 . Similarly we also decompose $C_{-}(0)$ in to $C_{-, 1} \cup C_{-, 2} \cup C_{-, 3}$, where $C_{-, 1}$ connects 0 and $u /(2 \pi)-\sqrt{-1}, C_{-, 2}$ connects $u /(2 \pi)-\sqrt{-1}$ and $1+u /(2 \pi)-\sqrt{-1}$, and $C_{-, 3}$ connects $1+u /(2 \pi)-\sqrt{-1}$ and -1 . Let $I_{ \pm, i}(N)$ be the integral of $(\tan (N \pi x)-\sqrt{-1}) g_{N}(x)$ along $C_{ \pm, i}(i=1,2,3)$. We 
will show that $\left|I_{ \pm, i}(N)\right|$ is bounded from above by $K_{ \pm, i} / N$ for a positive constant $K_{ \pm, i}$ independent of $N$.

We will give the following estimations for $I_{ \pm, i}(N)$.

$$
\begin{aligned}
& \left|I_{+, 1}(N)\right|<\frac{K_{+, 1}}{N} . \\
& \left|I_{+, 2}(N)\right|<\frac{K_{+, 2}}{N} . \\
& \left|I_{+, 3}(N)\right|<\frac{K_{+, 3}}{N} . \\
& \left|I_{-, 1}(N)\right|<\frac{K_{-, 1}}{N} . \\
& \left|I_{-, 2}(N)\right|<\frac{K_{-, 2}}{N} . \\
& \left|I_{-, 3}(N)\right|<\frac{K_{-, 3}}{N} .
\end{aligned}
$$

Proof of (6.3). We first estimate $|\tan (N \pi(-u /(2 \pi)+\sqrt{-1}) t)-\sqrt{-1}|$. We have

$$
\begin{aligned}
& \mid \tan (N \pi(-u /(2 \pi)+\sqrt{-1}) t))-\sqrt{-1} \mid \\
= & \left|\frac{2 e^{-N u t \sqrt{-1} / 2-N \pi t)}}{e^{-N u t \sqrt{-1} / 2-N \pi t)}+e^{N u t \sqrt{-1} / 2+N \pi t)}}\right| \\
= & \frac{2 e^{-2 N \pi t}}{\left|e^{-N u t \sqrt{-1}-2 N \pi t}+1\right|} .
\end{aligned}
$$

Since the denominator is bigger than 1 if $N u t<\pi / 2$ we have

$$
\mid \tan (N \pi(-u /(2 \pi)+\sqrt{-1}) t))-\sqrt{-1} \mid<2 e^{-2 N \pi t}
$$

when $t<\pi /(2 N u)$. If $t \geq \pi /(2 N u)$, then the denominator is bigger than equal to $1-e^{-2 N \pi t}$. So we have

$$
\mid \tan (N \pi(-u /(2 \pi)+\sqrt{-1}) t))-\sqrt{-1} \mid \leq \frac{2 e^{-2 N \pi t}}{1-e^{-2 N \pi t}} \leq \frac{2 e^{-2 N \pi t}}{1-e^{-\pi^{2} / u}}
$$

when $t \geq \pi /(2 N u)$. Therefore for any $0 \leq t \leq 1$ we have

$$
|\tan (N \pi(-u /(2 \pi)+\sqrt{-1}) t)-\sqrt{-1}|<\frac{2 e^{-2 N \pi t}}{1-e^{-\pi^{2} / u}} .
$$

So we have

$$
\left|I_{+, 1}(N)\right| \leq \frac{2}{1-e^{-\pi^{2} / u}} \int_{0}^{1} e^{-2 N \pi t}\left|g_{N}\left(\left(-\frac{u}{2 \pi}+\sqrt{-1}\right) t\right)\right| d t .
$$

We estimate $\left|g_{N}((-u /(2 \pi)+\sqrt{-1}) t)\right|$.

Since the function $g_{N}$ is not well-defined on the segment $(-u /(2 \pi)+\sqrt{-1}) t$ (Figure 11), we need to consider the segment $(-u /(2 \pi)+\sqrt{-1}) t+\varepsilon)(0 \leq t \leq 1)$ instead for small $\varepsilon$. (See the argument in [1, Page 534].) 
From (6.1) we have

$$
\begin{aligned}
& g_{N}(\left.\left(-\frac{u}{2 \pi}+\sqrt{-1}\right) t+\varepsilon\right) \\
&=\exp \left[N \Phi\left(\left(-\frac{u}{2 \pi}+\sqrt{-1}\right) t+\varepsilon\right)\right] \\
& \times \exp \left[I_{\gamma}\left(\pi-\sqrt{-1} u-\sqrt{-1} \frac{|\xi|^{2} t}{2 \pi}+\sqrt{-1} \varepsilon \xi\right)\right. \\
&\left.\quad-I_{\gamma}\left(-\pi-\sqrt{-1} u+\sqrt{-1} \frac{|\xi|^{2} t}{2 \pi}-\sqrt{-1} \varepsilon \xi\right)\right] .
\end{aligned}
$$

From Lemma 6.1, there exist $A>0$ and $B>0$ such that $\left|I_{\gamma}(z)\right| \leq 2 A+B|\gamma|(1+$ $\left.e^{-\operatorname{Im}(z) R}\right)$. So we have

$$
\begin{aligned}
& \left|g_{N}\left(\left(-\frac{u}{2 \pi}+\sqrt{-1}\right) t+\varepsilon\right)\right| \\
\leq & \exp \left[N \operatorname{Re} \Phi\left(\left(-\frac{u}{2 \pi}+\sqrt{-1}\right) t+\varepsilon\right)\right] \frac{\exp \left(2 A+B|\gamma|\left(1+e^{\left(u+|\xi|^{2} t /(2 \pi)-\varepsilon u\right) R}\right)\right.}{\exp \left(2 A+B|\gamma|\left(1+e^{\left(u-|\xi|^{2} t /(2 \pi)+\varepsilon u\right) R}\right)\right.} \\
\leq & \exp \left[N \operatorname{Re} \Phi\left(\left(-\frac{u t}{2 \pi}+\sqrt{-1}\right) t+\varepsilon\right)\right] \exp \left(2 A+B|\gamma|\left(1+e^{\left(u+|\xi|^{2} /(2 \pi)-\varepsilon u\right) R}\right)\right.
\end{aligned}
$$

for $0 \leq t \leq 1$.

Now we want to estimate $\operatorname{Re} \Phi((-u t /(2 \pi)+\sqrt{-1}) t+\varepsilon)$.

From the definition we have

$$
\begin{aligned}
& \Phi\left(\left(-\frac{u}{2 \pi}+\sqrt{-1}\right) t+\varepsilon\right) \\
& \quad=\frac{1}{\xi}\left(\operatorname{Li}_{2}\left(e^{u+|\xi|^{2} t /(2 \pi)-\varepsilon \xi}\right)-\operatorname{Li}_{2}\left(e^{u-|\xi|^{2} t /(2 \pi)+\varepsilon \xi}\right)\right)+\frac{u^{2} t}{2 \pi}-\sqrt{-1} u t-\varepsilon u .
\end{aligned}
$$

Since we may assume that $\operatorname{Re}\left(u+|\xi|^{2} t /(2 \pi)-\xi \varepsilon\right)=(1-\varepsilon) u+|\xi|^{2} t /(2 \pi)>0$, there are two cases to consider; the case where $u-|\xi|^{2} t /(2 \pi)<0$ and the case where $u-|\xi|^{2} t /(2 \pi) \geq 0$.

If $|z|>1$, it is convenient to replace $\operatorname{Li}_{2}(z)$ with $\operatorname{Li}_{2}\left(z^{-1}\right)$ using the following well-known formula.

$$
\operatorname{Li}_{2}(z)+\operatorname{Li}_{2}\left(z^{-1}\right)=-\frac{\pi^{2}}{6}-\frac{1}{2}(\log (-z))^{2}
$$

where we choose a branch of $\log (-z)$ so that $-\pi<\operatorname{Im} \log (-z)<\pi$. 
- The case where $u-|\xi|^{2} t /(2 \pi)<0$. We choose $\varepsilon$ small enough so that $u-|\xi|^{2} t /(2 \pi)+\varepsilon u<0$. From (6.12) we have

$$
\begin{aligned}
& \Phi\left(\left(-\frac{u}{2 \pi}+\sqrt{-1}\right) t+\varepsilon\right) \\
& =\frac{1}{\xi}\left(-\operatorname{Li}_{2}\left(e^{-u-|\xi|^{2} t /(2 \pi)+\varepsilon \xi}\right)-\operatorname{Li}_{2}\left(e^{u-|\xi|^{2} t /(2 \pi)+\varepsilon \xi}\right)\right. \\
& \left.-\frac{\pi^{2}}{6}-\frac{1}{2}\left(u+\frac{|\xi|^{2} t}{2 \pi}-\varepsilon \xi+\sqrt{-1} \pi\right)^{2}\right) \\
& +\frac{u^{2} t}{2 \pi}-\sqrt{-1} u t-\varepsilon u \\
& =\frac{1}{\xi}\left(-\operatorname{Li}_{2}\left(e^{u-|\xi|^{2} t /(2 \pi)+\xi \varepsilon}\right)-\operatorname{Li}_{2}\left(e^{u-|\xi|^{2} t /(2 \pi)+\xi \varepsilon}\right)\right) \\
& -\frac{\pi^{2}}{6 \xi}-\frac{1}{2 \xi}\left(u+\frac{|\xi|^{2} t}{2 \pi}-\varepsilon \xi+\sqrt{-1} \pi\right)^{2} \\
& +\frac{u^{2} t}{2 \pi}-\sqrt{-1} u t-\varepsilon u,
\end{aligned}
$$

where in the first equality we choose the sign of $\sqrt{-1} \pi$ so that $\operatorname{Im}(u+$ $\left.|\xi|^{2} t /(2 \pi)-\varepsilon \xi+\pi \sqrt{-1}\right)=-\varepsilon u+\pi$ is between $-\pi$ and $\pi$. Since the dilogarithm function $\operatorname{Li}(z)$ is analytic when $\operatorname{Re}(z)<1$, we have

$$
\begin{aligned}
& \lim _{\varepsilon \searrow 0} \Phi\left(\left(-\frac{u}{2 \pi}+\sqrt{-1}\right) t+\varepsilon\right) \\
= & \frac{1}{\xi}\left(-\operatorname{Li}_{2}\left(e^{-u-|\xi|^{2} t /(2 \pi)}\right)-\operatorname{Li}_{2}\left(e^{u-|\xi|^{2} t /(2 \pi)}\right)\right) \\
& -\frac{\pi^{2}}{6 \xi}-\frac{1}{2 \xi}\left(u+\frac{|\xi|^{2} t}{2 \pi}+\sqrt{-1} \pi\right)^{2}+\frac{u^{2} t}{2 \pi}-\sqrt{-1} u t .
\end{aligned}
$$

Since $\operatorname{Li}_{2}(z)$ is real when $z$ is real and $z<1$, we have

$$
\begin{aligned}
& \lim _{\varepsilon \searrow 0} \operatorname{Re} \Phi\left(\left(-\frac{u}{2 \pi}+\sqrt{-1}\right) t+\varepsilon\right) \\
= & -\frac{u}{|\xi|^{2}}\left(\operatorname{Li}_{2}\left(e^{-u-|\xi|^{2} t /(2 \pi)}\right)+\operatorname{Li}_{2}\left(e^{u-|\xi|^{2} t /(2 \pi)}\right)\right) \\
& -\frac{u \pi^{2}}{6|\xi|^{2}}-\frac{u}{2|\xi|^{2}}\left(u+\frac{|\xi|^{2} t}{2 \pi}\right)^{2}+\frac{u \pi^{2}}{2|\xi|^{2}}-\frac{4 \pi\left(u+|\xi|^{2} t /(2 \pi)\right) \pi}{2|\xi|^{2}}+\frac{u^{2} t}{2 \pi} \\
= & -\frac{u}{|\xi|^{2}}\left(\operatorname{Li}_{2}\left(e^{-u-|\xi|^{2} t /(2 \pi)}\right)+\operatorname{Li}_{2}\left(e^{u-|\xi|^{2} t /(2 \pi)}\right)\right) \\
& -\frac{5 u \pi^{2}}{6|\xi|^{2}}-\frac{u^{3}}{2|\xi|^{2}}-\frac{u|\xi|^{2} t^{2}}{8 \pi^{2}}-\pi t \\
< & 0
\end{aligned}
$$

if $u>0$. 
- The case where $u-|\xi|^{2} t /(2 \pi) \geq 0$. In this case we have

$$
\begin{aligned}
& \Phi\left(\left(-\frac{u}{2 \pi}+\sqrt{-1}\right) t+\varepsilon\right) \\
& =\frac{1}{\xi}\left(-\mathrm{Li}_{2}\left(e^{-u-|\xi|^{2} t /(2 \pi)+\varepsilon \xi}\right)+\operatorname{Li}_{2}\left(e^{-u+|\xi|^{2} t /(2 \pi)-\varepsilon \xi}\right)\right. \\
& \left.-\frac{1}{2}\left(u+\frac{|\xi|^{2} t}{2 \pi}-\varepsilon \xi+\pi \sqrt{-1}\right)^{2}+\frac{1}{2}\left(u-\frac{|\xi|^{2} t}{2 \pi}+\varepsilon \xi-\pi \sqrt{-1}\right)^{2}\right) \\
& +\frac{u^{2} t}{2 \pi}-\sqrt{-1} u t-u \varepsilon \\
& =\frac{1}{\xi}\left(-\operatorname{Li}_{2}\left(e^{-u-|\xi|^{2} t /(2 \pi)+\varepsilon \xi}\right)+\operatorname{Li}_{2}\left(e^{-u+|\xi|^{2} t /(2 \pi)-\varepsilon \xi}\right)\right) \\
& -\frac{2 u}{\xi}\left(\frac{|\xi|^{2} t}{2 \pi}-\varepsilon \xi+\pi \sqrt{-1}\right)+\frac{u^{2} t}{2 \pi}-\sqrt{-1} u t-u \varepsilon
\end{aligned}
$$

and so we have

$$
\begin{aligned}
& \lim _{\varepsilon \searrow 0} \operatorname{Re} \Phi\left(\left(-\frac{u}{2 \pi}+\sqrt{-1}\right) t+\varepsilon\right) \\
= & \frac{u}{|\xi|^{2}}\left(-\operatorname{Li}_{2}\left(e^{-u-|\xi|^{2} t /(2 \pi)}\right)+\operatorname{Li}_{2}\left(e^{-u+|\xi|^{2} t /(2 \pi)}\right)\right) \\
& -\operatorname{Re}\left(\frac{2 u}{\xi}\left(\frac{|\xi|^{2} t}{2 \pi}+\pi \sqrt{-1}\right)\right)+\frac{u^{2} t}{2 \pi} \\
= & \frac{u}{|\xi|^{2}}\left(-\operatorname{Li}_{2}\left(e^{-u-|\xi|^{2} t /(2 \pi)}\right)+\operatorname{Li}_{2}\left(e^{-u+|\xi|^{2} t /(2 \pi)}\right)\right)-\frac{4 \pi^{2} u}{|\xi|^{2}}-\frac{u^{2} t}{2 \pi} \\
< & 0
\end{aligned}
$$

if $u>0$.

Therefore for any $t$ we have $\operatorname{Re} \Phi((-u /(2 \pi)+\sqrt{-1}) t+\varepsilon) \leq 0$ for small $\varepsilon>0$.

So from (6.10) and (6.11) we have

$$
\begin{aligned}
\left|I_{+, 1}(N)\right| & \leq \frac{2}{1-e^{-\pi^{2} / u}} \exp \left(2 A+B|\gamma|\left(1+e^{\left(u+|\xi|^{2} /(2 \pi)-\varepsilon u\right) R}\right) \int_{0}^{1} e^{-2 N \pi t} d t\right. \\
& =\frac{1-e^{-2 N \pi}}{N \pi\left(1-e^{-\pi^{2} / u}\right)} \exp \left(2 A+B|\gamma|\left(1+e^{\left(u+|\xi|^{2} /(2 \pi)\right) R}\right)\right. \\
& <\frac{K_{+, 1}}{N}
\end{aligned}
$$

for a positive constant $K_{+, 1}$.

Proof of (6.6). Since tan is an odd function we have from (6.9)

$$
|\tan (N \pi(u /(2 \pi)-\sqrt{-1}) t)+\sqrt{-1}|<\frac{2 e^{-2 N \pi t}}{1-e^{-\pi^{2} / u}}
$$

for any $0 \leq t \leq 1$.

So we have

$$
\left|I_{-, 1}(N)\right| \leq \frac{2}{1-e^{-\pi^{2} / u}} \int_{0}^{1} e^{-2 N \pi t}\left|g_{N}\left(\left(\frac{u}{2 \pi}-\sqrt{-1}\right) t\right)\right| d t .
$$

We estimate $\left|g_{N}((u /(2 \pi)-\sqrt{-1}) t)\right|$.

As in the case of $I_{+, 1}$ we need to calculate $g_{N}\left(g_{N}((u /(2 \pi)-\sqrt{-1}) t)+\varepsilon\right)$. 
From (6.1) we have

$$
\begin{aligned}
& g_{N}(\left.\left(\frac{u}{2 \pi}-\sqrt{-1}\right) t+\varepsilon\right) \\
&=\exp \left[N \Phi\left(\left(\frac{u}{2 \pi}-\sqrt{-1}\right) t+\varepsilon\right)\right] \\
& \times \exp \left[I_{\gamma}\left(\pi-\sqrt{-1} u+\sqrt{-1} \frac{|\xi|^{2} t}{2 \pi}+\sqrt{-1} \varepsilon \xi\right)\right. \\
&\left.\quad-I_{\gamma}\left(-\pi-\sqrt{-1} u-\sqrt{-1} \frac{|\xi|^{2} t}{2 \pi}-\sqrt{-1} \varepsilon \xi\right)\right] .
\end{aligned}
$$

From Lemma 6.1, there exist $A>0$ and $B>0$ such that $\left|I_{\gamma}(z)\right| \leq 2 A+B|\gamma|(1+$ $\left.e^{-\operatorname{Im}(z) R}\right)$. So we have

$$
\begin{aligned}
& \left|g_{N}\left(\left(\frac{u}{2 \pi}-\sqrt{-1}\right) t+\varepsilon\right)\right| \\
\leq & \exp \left[N \operatorname{Re} \Phi\left(\left(\frac{u}{2 \pi}-\sqrt{-1}\right) t+\varepsilon\right)\right] \frac{\exp \left(2 A+B|\gamma|\left(1+e^{\left(u-|\xi|^{2} t /(2 \pi)-\varepsilon u\right) R}\right)\right.}{\exp \left(2 A+B|\gamma|\left(1+e^{\left(u+|\xi|^{2} t /(2 \pi)+\varepsilon u\right) R}\right)\right.} \\
\leq & \exp \left[N \operatorname{Re} \Phi\left(\left(\frac{u t}{2 \pi}-\sqrt{-1}\right) t+\varepsilon\right)\right] \exp \left(2 A+B|\gamma|\left(1+e^{\left(u-|\xi|^{2} /(2 \pi)-\varepsilon u\right) R}\right)\right.
\end{aligned}
$$

for $0 \leq t \leq 1$.

Now we want to estimate $\operatorname{Re} \Phi((u t /(2 \pi)-\sqrt{-1}) t+\varepsilon)$.

From the definition we have

$$
\begin{aligned}
& \Phi\left(\left(\frac{u}{2 \pi}-\sqrt{-1}\right) t+\varepsilon\right) \\
& \quad=\frac{1}{\xi}\left(\operatorname{Li}_{2}\left(e^{u-|\xi|^{2} t /(2 \pi)-\varepsilon \xi}\right)-\operatorname{Li}_{2}\left(e^{u+|\xi|^{2} t /(2 \pi)+\varepsilon \xi}\right)\right)-\frac{u^{2} t}{2 \pi}+\sqrt{-1} u t-\varepsilon u .
\end{aligned}
$$

Since $\operatorname{Re}\left(u+|\xi|^{2} t /(2 \pi)+\varepsilon \xi\right)=(1+\varepsilon) u+|\xi|^{2} t /(2 \pi)>0$, there are two cases to consider; the case where $u-|\xi|^{2} t /(2 \pi)<0$ and the case where $u-|\xi|^{2} t /(2 \pi) \geq 0$.

- The case where $u-|\xi|^{2} t /(2 \pi) \leq 0$. In this case we have $u-|\xi|^{2} t /(2 \pi)-\varepsilon u<$ 0 . From (6.12) we have

$$
\begin{aligned}
& \Phi\left(\left(\frac{u}{2 \pi}-\sqrt{-1}\right) t+\varepsilon\right) \\
= & \frac{1}{\xi}\left(\operatorname{Li}_{2}\left(e^{u-|\xi|^{2} t /(2 \pi)-\varepsilon \xi}\right)+\operatorname{Li}_{2}\left(e^{-u-|\xi|^{2} t /(2 \pi)-\varepsilon \xi}\right)\right. \\
& \left.+\frac{\pi^{2}}{6}+\frac{1}{2}\left(u+\frac{|\xi|^{2} t}{2 \pi}+\varepsilon \xi-\sqrt{-1} \pi\right)^{2}\right) \\
& -\frac{u^{2} t}{2 \pi}+\sqrt{-1} u t-\varepsilon u \\
= & \frac{1}{\xi}\left(\operatorname{Li}_{2}\left(e^{u-|\xi|^{2} t /(2 \pi)-\xi \varepsilon}\right)+\operatorname{Li}_{2}\left(e^{-u-|\xi|^{2} t /(2 \pi)-\xi \varepsilon}\right)\right) \\
& +\frac{\pi^{2}}{6 \xi}+\frac{1}{2 \xi}\left(u+\frac{|\xi|^{2} t}{2 \pi}+\varepsilon \xi-\sqrt{-1} \pi\right)^{2} \\
& -\frac{u^{2} t}{2 \pi}+\sqrt{-1} u t-\varepsilon u .
\end{aligned}
$$


Therefore we have

$$
\begin{aligned}
& \lim _{\varepsilon \searrow 0} \Phi\left(\left(\frac{u}{2 \pi}-\sqrt{-1}\right) t+\varepsilon\right) \\
= & \frac{1}{\xi}\left(\operatorname{Li}_{2}\left(e^{u-|\xi|^{2} t /(2 \pi)}\right)+\operatorname{Li}_{2}\left(e^{-u-|\xi|^{2} t /(2 \pi)}\right)\right) \\
& +\frac{\pi^{2}}{6 \xi}+\frac{1}{2 \xi}\left(u+\frac{|\xi|^{2} t}{2 \pi}-\sqrt{-1} \pi\right)^{2} \\
& -\frac{u^{2} t}{2 \pi}+\sqrt{-1} u t
\end{aligned}
$$

and so

$$
\begin{aligned}
& \lim _{\varepsilon \searrow 0} \operatorname{Re} \Phi\left(\left(\frac{u}{2 \pi}-\sqrt{-1}\right) t+\varepsilon\right) \\
= & \frac{u}{|\xi|^{2}}\left(\operatorname{Li}_{2}\left(e^{u-|\xi|^{2} t /(2 \pi)}\right)+\operatorname{Li}_{2}\left(e^{-u-|\xi|^{2} t /(2 \pi)}\right)\right) \\
& +\frac{u \pi^{2}}{6|\xi|^{2}}+\frac{u}{2|\xi|^{2}}\left(u+\frac{|\xi|^{2} t}{2 \pi}\right)^{2}-\frac{u \pi^{2}}{2|\xi|^{2}}-\frac{4 \pi\left(u+|\xi|^{2} t /(2 \pi)\right) \pi}{2|\xi|^{2}}-\frac{u^{2} t}{2 \pi} \\
= & \frac{u}{|\xi|^{2}}\left(\operatorname{Li}_{2}\left(e^{u-|\xi|^{2} t /(2 \pi)}\right)+\operatorname{Li}_{2}\left(e^{-u-|\xi|^{2} t /(2 \pi)}\right)\right) \\
& -\frac{7 u \pi^{2}}{3|\xi|^{2}}+\frac{u^{3}}{2|\xi|^{2}}+\frac{u|\xi|^{2} t^{2}}{8 \pi^{2}}-\pi t \\
= & -2 \frac{u \pi^{2}}{|\xi|^{2}}+\frac{u^{3}}{2|\xi|^{2}}+\frac{u|\xi|^{2} t^{2}}{8 \pi^{2}}-\pi t
\end{aligned}
$$

since $\operatorname{Li}_{2}(z) \leq \pi^{2} / 6$ for $0<z \leq 1$. We can easily prove

$$
-2 \frac{u \pi^{2}}{|\xi|^{2}}+\frac{u^{3}}{2|\xi|^{2}}+\frac{u|\xi|^{2} t^{2}}{8 \pi^{2}}-\pi t<\pi t
$$

when $0<u<1$ and $2 u \pi /|\xi|^{2} \leq t \leq 1$. So we have

$$
\lim _{\varepsilon \searrow 0} \operatorname{Re} \Phi\left(\left(\frac{u}{2 \pi}-\sqrt{-1}\right) t+\varepsilon\right)<\pi t
$$

in this case.

- The case where $u-|\xi|^{2} t /(2 \pi)>0$. We choose $\varepsilon$ so that $u-|\xi|^{2} t /(2 \pi)-\varepsilon u>$ 0 . In this case we have

$$
\begin{aligned}
& \Phi( \\
=\frac{1}{\xi}( & (-\sqrt{-1}) t+\varepsilon) \\
& \left.-\frac{1}{2}\left(u-\frac{|\xi|^{2} t}{2 \pi}-\varepsilon \xi+\pi \sqrt{-1}\right)^{2}+\frac{1}{2}\left(-u-\frac{|\xi|^{2} t}{2 \pi}-\varepsilon \xi+\pi \sqrt{-1}\right)^{2}\right) \\
- & \frac{u^{2} t}{2 \pi}+\sqrt{-1} u t-\varepsilon u \\
\text { and } \operatorname{so} \text { we have } & \\
& \quad \lim ^{2} \operatorname{Re} \Phi\left(\left(\frac{u}{2 \pi}-\sqrt{-1}\right) t+\varepsilon\right) \\
= & \frac{u}{|\xi|^{2}}\left(-\operatorname{Li}_{2}\left(e^{-u+|\xi|^{2} t /(2 \pi)}\right)+\operatorname{Li}_{2}\left(e^{-u-|\xi|^{2} t /(2 \pi)}\right)\right)+\frac{u^{2} t}{2 \pi}-\frac{4 u \pi^{2}}{|\xi|^{2}} \\
< & \frac{u^{2} t}{2 \pi}-\frac{23 u \pi^{2}}{6|\xi|^{2}}<0
\end{aligned}
$$




$$
\text { if } 0<u<1 \text { and } 0 \leq t<2 u \pi /|\xi|^{2} \text {. }
$$

Therefore for any $0 \leq t \leq 1$ we have $\operatorname{Re} \Phi((u /(2 \pi)-\sqrt{-1}) t+\varepsilon) \leq \pi t$ for small $\varepsilon>0$.

So from (6.14) and (6.15) we have

$$
\begin{aligned}
\left|I_{-, 1}(N)\right| & \leq \frac{2}{1-e^{-\pi^{2} / u}} \exp \left(2 A+B|\gamma|\left(1+e^{\left(u-|\xi|^{2} /(2 \pi)\right) R}\right) \int_{0}^{1} e^{-N \pi t} d t\right. \\
& =\frac{2\left(1-e^{-N \pi}\right)}{N\left(1-e^{-\pi^{2} / u}\right)} \exp \left(2 A+B|\gamma|\left(1+e^{\left(u-|\xi|^{2} /(2 \pi)\right) R}\right)\right. \\
& <\frac{K_{-, 1}}{N}
\end{aligned}
$$

for a positive constant $K_{-, 1}$.

Proof of (6.5). Since tan has period $\pi$ we have

$$
|\tan (N \pi(-u /(2 \pi)+\sqrt{-1}) t)-\sqrt{-1}|<\frac{2 e^{-2 N \pi t}}{1-e^{-\pi^{2} / u}}
$$

from 6.9 )

From (6.16) we have

$$
\left|I_{+, 3}(N)\right| \leq \frac{2}{1-e^{-\pi^{2} / u}} \int_{0}^{1} e^{-2 N \pi t}\left|g_{N}\left(\left(-\frac{u}{2 \pi}+\sqrt{-1}\right) t+1\right)\right| d t .
$$

As in the case of $I_{+, 1}(N)$ we consider the integral on the segment $(-u /(2 \pi)+$ $\sqrt{-1}) t+1-\varepsilon(0 \leq t \leq 1)$ for small $\varepsilon$.

We estimate $\Phi((-u /(2 \pi)+\sqrt{-1}) t+1-\varepsilon)$. We have

$$
\begin{aligned}
& \Phi\left(\left(-\frac{u}{2 \pi}+\sqrt{-1}\right) t+1-\varepsilon\right) \\
= & \frac{1}{\xi}\left(\operatorname{Li}_{2}\left(e^{u+|\xi|^{2} t /(2 \pi)-(1-\varepsilon) \xi}\right)-\operatorname{Li}_{2}\left(e^{u-|\xi|^{2} t /(2 \pi)+(1-\varepsilon) \xi}\right)\right) \\
& +\frac{u^{2} t}{2 \pi}-\sqrt{-1} u t-(1-\varepsilon) u .
\end{aligned}
$$

Since we may assume that $\operatorname{Re}\left(u+|\xi|^{2} t /(2 \pi)-(1-\varepsilon) \xi\right)=\varepsilon u+|\xi|^{2} t /(2 \pi)>0$, there are two cases to consider; the case where $2 u-|\xi|^{2} t /(2 \pi) \leq 0$ and the case where $2 u-|\xi|^{2} t /(2 \pi)>0$.

- $2 u-|\xi|^{2} t /(2 \pi) \leq 0$. In this case, since $u-|\xi|^{2} t /(2 \pi)+(1-\varepsilon) u<0$, from 6.12) we have

$$
\begin{aligned}
& \Phi\left(\left(-\frac{u}{2 \pi}+\sqrt{-1}\right) t+1-\varepsilon\right) \\
= & \frac{1}{\xi}\left(-\operatorname{Li}_{2}\left(e^{-u-|\xi|^{2} t /(2 \pi)+(1-\varepsilon) \xi}\right)-\operatorname{Li}_{2}\left(e^{u-|\xi|^{2} t /(2 \pi)+(1-\varepsilon) \xi}\right)\right. \\
& \left.\quad-\frac{\pi^{2}}{6}-\frac{1}{2}\left(u+\frac{|\xi|^{2} t}{2 \pi}-(1-\varepsilon) \xi+\pi \sqrt{-1}\right)^{2}\right) \\
+ & \frac{u^{2} t}{2 \pi}-\sqrt{-1} u t-(1-\varepsilon) u
\end{aligned}
$$


Since the dilogarithm function $\operatorname{Li}(z)$ is analytic when $\operatorname{Re}(z)<1$, we have

$$
\begin{aligned}
& \lim _{\varepsilon \searrow 0} \Phi\left(\left(-\frac{u}{2 \pi}+\sqrt{-1}\right) t+1-\varepsilon\right) \\
= & \frac{1}{\xi}\left(-\operatorname{Li}_{2}\left(e^{-|\xi|^{2} t /(2 \pi)}\right)-\operatorname{Li}_{2}\left(e^{2 u-|\xi|^{2} t /(2 \pi)}\right)\right) \\
& -\frac{\pi^{2}}{6 \xi}-\frac{1}{2 \xi}\left(\frac{|\xi|^{2} t}{2 \pi}-\pi \sqrt{-1}\right)^{2} \\
& +\frac{u^{2} t}{2 \pi}-\sqrt{-1} u t-u .
\end{aligned}
$$

So we have

$$
\begin{aligned}
& \lim _{\varepsilon \searrow 0} \operatorname{Re}\left(\left(-\frac{u}{2 \pi}+\sqrt{-1}\right) t+1-\varepsilon\right) \\
= & -\frac{u}{|\xi|^{2}}\left(\operatorname{Re~Li}_{2}\left(e^{-|\xi|^{2} t /(2 \pi)}\right)+\operatorname{Re~Li}_{2}\left(e^{2 u-|\xi|^{2} t /(2 \pi)}\right)\right) \\
& -\frac{u \pi^{2}}{6|\xi|^{2}}-\frac{u}{2|\xi|^{2}} \frac{|\xi|^{4} t^{2}}{4 \pi^{2}}+\frac{u \pi^{2}}{2|\xi|^{2}}-\frac{4 \pi|\xi|^{2} t /(2 \pi) \pi}{2|\xi|^{2}}+\frac{u^{2} t}{2 \pi}-u \\
= & -\frac{u}{|\xi|^{2}}\left(\operatorname{Re} \operatorname{Li}_{2}\left(e^{-|\xi|^{2} t /(2 \pi)}\right)+\operatorname{Re}_{2}\left(e^{2 u-|\xi|^{2} t /(2 \pi)}\right)\right) \\
& +\frac{u \pi^{2}}{3|\xi|^{2}}-\frac{u|\xi|^{2} t^{2}}{8 \pi^{2}}-\pi t+\frac{u^{2} t}{2 \pi}-u \leq 0 .
\end{aligned}
$$

The last inequality follows since $\frac{u \pi^{2}}{3|\xi|^{2}}-\frac{u|\xi|^{2} t^{2}}{8 \pi^{2}}-\pi t+\frac{u^{2} t}{2 \pi}-u$, is a quadratic function with respect to $u$ with non-positive maximum.

- $2 u-|\xi|^{2} t /(2 \pi)>0$. In this case we may choose $\varepsilon$ small so that $u-$ $|\xi|^{2} t /(2 \pi)+(1-\varepsilon) u>0$. Then we have

$$
\begin{aligned}
& \Phi\left(\left(-\frac{u}{2 \pi}+\sqrt{-1}\right) t+1-\varepsilon\right) \\
= & \frac{1}{\xi}\left(-\operatorname{Li}_{2}\left(e^{-u-|\xi|^{2} t /(2 \pi)+(1-\varepsilon) \xi}\right)+\operatorname{Li}_{2}\left(e^{-u+|\xi|^{2} t /(2 \pi)-(1-\varepsilon) \xi}\right)\right. \\
& \left.-\frac{1}{2}\left(u+\frac{|\xi|^{2} t}{2 \pi}-(1-\varepsilon) \xi+\pi \sqrt{-1}\right)^{2}+\frac{1}{2}\left(u-\frac{|\xi|^{2} t}{2 \pi}+(1-\varepsilon) \xi-\pi \sqrt{-1}\right)^{2}\right) \\
& +\frac{u^{2} t}{2 \pi}-\sqrt{-1} u t-(1-\varepsilon) u
\end{aligned}
$$

and so we have

$$
\begin{aligned}
& \lim _{\varepsilon \searrow 0} \operatorname{Re} \Phi\left(\left(-\frac{u}{2 \pi}+\sqrt{-1}\right) t+1-\varepsilon\right) \\
= & \frac{u}{|\xi|^{2}}\left(-\operatorname{Li}_{2}\left(e^{-|\xi|^{2} t /(2 \pi)}\right)+\operatorname{Li}_{2}\left(e^{-2 u+|\xi|^{2} t /(2 \pi)}\right)\right)+\frac{2 u^{3}}{|\xi|^{2}}-\frac{u^{2} t}{2 \pi}+\frac{4 \pi^{2} u}{|\xi|^{2}}-u .
\end{aligned}
$$

Putting $s:=|\xi|^{2} t /(2 \pi)$ and consider the function

$$
\begin{aligned}
f(u, s) & :=-\operatorname{Li}_{2}\left(e^{-s}\right)+\operatorname{Li}_{2}\left(e^{-2 u+s}\right)+2 u^{2}-u s+4 \pi^{2}-|\xi|^{2}-\frac{2 \pi^{2} s}{u} \\
& =-\operatorname{Li}_{2}\left(e^{-s}\right)+\operatorname{Li}_{2}\left(e^{-2 u+s}\right)+u^{2}-u s-\frac{2 \pi^{2} s}{u}
\end{aligned}
$$

so that

$$
\frac{u}{|\xi|^{2}} f\left(u, \frac{|\xi|^{2} t}{2 \pi}\right)=\lim _{\varepsilon \searrow 0} \operatorname{Re} \Phi\left(\left(-\frac{u}{2 \pi}+\sqrt{-1}\right) t+1-\varepsilon\right)-\pi t .
$$


We will show $f(u, s) \leq 0$ for $0 \leq s<2 u \leq 2$.

We have

$$
\exp \left[-\frac{\partial f(u, t)}{\partial t}\right]=2 e^{2 \pi^{2} / u}(\cosh (u)-\cosh (s-u)) .
$$

Therefore it can be shown that for fixed $u, f(u, s)$ is increasing for $0 \leq s<$ $u-\operatorname{arccosh}\left(\cosh (u)-\exp \left(-2 \pi^{2} / u\right) / 2\right)$, decreasing for $u-\operatorname{arccosh}(\cosh (u)-$ $\left.\exp \left(-2 \pi^{2} / u\right) / 2\right)<s<u+\operatorname{arccosh}\left(\cosh (u)-\exp \left(-2 \pi^{2} / u\right) / 2\right)$, and increasing for $u+\operatorname{arccosh}\left(\cosh (u)-\exp \left(-2 \pi^{2} / u\right) / 2\right)<s<u$. Since a graph of $f\left(u, u-\operatorname{arccosh}\left(\cosh (u)-\exp \left(-2 \pi^{2} / u\right) / 2\right)\right)$ looks as Figure 4 and

$$
f(u, 2 u)=-\frac{23 \pi^{2}}{6}-u^{2}-\operatorname{Li}_{2}\left(e^{-2 u}\right)<0,
$$

we see $f(u, s) \leq 0$.

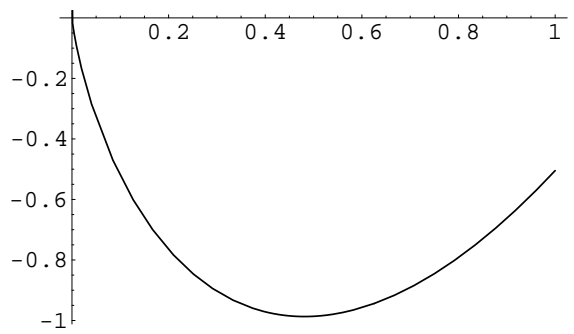

Figure 4. A graph of $f\left(u, u-\operatorname{arccosh}\left(\cosh (u)-\exp \left(-4 \pi^{2} / u\right) / 2\right)\right)$.

Therefore we finally have

$$
\begin{aligned}
\left|I_{+, 3}(N)\right| & <\frac{2}{1-e^{-\pi^{2} / u}} \exp \left(2 A+B\left(1+e^{\left(u+|\xi|^{2} /(2 \pi)\right) R}\right)|\gamma|\right) \int_{4 \pi u /|\xi|^{2}}^{1} e^{-2 N \pi t} d t \\
& =\frac{e^{-8 N \pi^{2} u /|\xi|^{2}}-e^{-2 N \pi}}{N \pi\left(1-e^{-\pi^{2} / u}\right)} \exp \left(2 A+B\left(1+e^{\left(u+|\xi|^{2} /(2 \pi)\right) R}\right)|\gamma|\right) \\
& <\frac{K_{+, 3}}{N}
\end{aligned}
$$

for a positive constant $K_{+, 3}$.

Proof of (6.8). Since tan has period $\pi$ we have

$$
\mid \tan \left(N \pi((u /(2 \pi)-\sqrt{-1}) t+1)+\sqrt{-1} \mid<\frac{2 e^{-2 N \pi t}}{1-e^{-\pi^{2} / u}}\right.
$$

from 6.9

From (6.18) we have

$$
\left|I_{-, 3}(N)\right| \leq \frac{2}{1-e^{-\pi^{2} / u}} \int_{0}^{1} e^{-2 N \pi t}\left|g_{N}\left(\left(-\frac{u}{2 \pi}+\sqrt{-1}\right) t+1\right)\right| d t .
$$

As in the case of $I_{+, 1}(N)$ we consider the integral on the segment $(u /(2 \pi)-$ $\sqrt{-1}) t+1-\varepsilon(0 \leq t \leq 1)$ for small $\varepsilon$.

We estimate $\Phi((u /(2 \pi)-\sqrt{-1}) t+1-\varepsilon)$. We have

$$
\begin{aligned}
& \Phi\left(\left(\frac{u}{2 \pi}-\sqrt{-1}\right) t+1-\varepsilon\right) \\
= & \frac{1}{\xi}\left(\operatorname{Li}_{2}\left(e^{u-|\xi|^{2} t /(2 \pi)-(1-\varepsilon) \xi}\right)-\operatorname{Li}_{2}\left(e^{u+|\xi|^{2} t /(2 \pi)+(1-\varepsilon) \xi}\right)\right) \\
& -\frac{u^{2} t}{2 \pi}+\sqrt{-1} u t-(1-\varepsilon) u .
\end{aligned}
$$


We will calculate $\lim _{\varepsilon \searrow 0} \Phi((u /(2 \pi)-\sqrt{-1}) t+1-\varepsilon)$.

Note that $\operatorname{Re}\left(u+|\xi|^{2} t /(2 \pi)+(1-\varepsilon) \xi\right)=2 u+|\xi|^{2} t /(2 \pi)-\varepsilon u>0$ for small $\varepsilon$. Since $\operatorname{Re}\left(u-|\xi|^{2} t /(2 \pi)-(1-\varepsilon) \xi\right)=-|\xi|^{2} t /(2 \pi)+\varepsilon u$, if $t>0$ we assume that $\operatorname{Re}\left(u-|\xi|^{2} t /(2 \pi)-(1-\varepsilon) \xi\right)<0$. Therefore we assume that $t>0$.

In this case from 6.12) we have

$$
\begin{aligned}
& \Phi\left(\left(\frac{u}{2 \pi}-\sqrt{-1}\right) t+1-\varepsilon\right) \\
= & \frac{1}{\xi}\left(\operatorname{Li}_{2}\left(e^{-|\xi|^{2} t /(2 \pi)+\varepsilon \xi}\right)+\operatorname{Li}_{2}\left(e^{-2 u-|\xi|^{2} t /(2 \pi)+\varepsilon \xi}\right)\right) \\
& +\frac{\pi^{2}}{6 \xi}+\frac{1}{2 \xi}\left(2 u+\frac{|\xi|^{2} t}{2 \pi}-\varepsilon \xi+\pi \sqrt{-1}\right)^{2} \\
& -\frac{u^{2} t}{2 \pi}+\sqrt{-1} u t-(1-\varepsilon) u
\end{aligned}
$$

and

$$
\begin{aligned}
& \operatorname{Re} \Phi\left(\left(\frac{u}{2 \pi}-\sqrt{-1}\right) t+1-\varepsilon\right) \\
= & \frac{1}{\xi}\left(\operatorname{Li}_{2}\left(e^{-|\xi|^{2} t /(2 \pi)+\varepsilon \xi}\right)+\operatorname{Li}_{2}\left(e^{-2 u-|\xi|^{2} t /(2 \pi)+\varepsilon \xi}\right)\right) \\
& +\frac{\pi^{2}}{6 \xi}+\frac{1}{2 \xi}\left(2 u+\frac{|\xi|^{2} t}{2 \pi}-\varepsilon \xi+\pi \sqrt{-1}\right)^{2} \\
& -\frac{u^{2} t}{2 \pi}+\sqrt{-1} u t-(1-\varepsilon) u .
\end{aligned}
$$

So we have

$$
\begin{aligned}
& \lim _{\varepsilon \searrow 0} \Phi\left(\left(\frac{u}{2 \pi}-\sqrt{-1}\right) t+1-\varepsilon\right) \\
= & \frac{1}{\xi}\left(\operatorname{Li}_{2}\left(e^{-|\xi|^{2} t /(2 \pi)}\right)+\operatorname{Li}_{2}\left(e^{-2 u-|\xi|^{2} t /(2 \pi)}\right)\right) \\
& +\frac{\pi^{2}}{6 \xi}+\frac{1}{2 \xi}\left(2 u+\frac{|\xi|^{2} t}{2 \pi}+\pi \sqrt{-1}\right)^{2} \\
& -\frac{u^{2} t}{2 \pi}+\sqrt{-1} u t-u \\
= & \frac{u}{|\xi|^{2}}\left(\operatorname{Li}_{2}\left(e^{-|\xi|^{2} t /(2 \pi)}\right)+\operatorname{Li}_{2}\left(e^{-2 u-|\xi|^{2} t /(2 \pi)}\right)\right) \\
& +\frac{u \pi^{2}}{\left.6\right|^{2}}+\frac{u}{2|\xi|^{2}}\left(2 u+\frac{|\xi|^{2} t}{2 \pi}\right)^{2}-\frac{u \pi^{2}}{2|\xi|^{2}}+\frac{4 \pi^{2}}{2|\xi|^{2}}\left(2 u+\frac{|\xi|^{2} t}{2 \pi}\right)-\frac{u^{2} t}{2 \pi}-u \\
\leq & \frac{11 u \pi^{2}}{3|\xi|^{2}}+\frac{2 u^{3}}{|\xi|^{2}}+\frac{u|\xi|^{2} t^{2}}{8 \pi^{2}}+\pi t+\frac{u^{2} t}{2 \pi}-u<\frac{3}{2} \pi t
\end{aligned}
$$

if $0<u<1$ and $0<t \leq 1$.

Therefore we finally have

$$
\begin{aligned}
\left|I_{-, 3}(N)\right| & <\frac{2}{1-e^{-\pi^{2} / u}} \exp \left(2 A+B\left(1+e^{\left(u+|\xi|^{2} /(2 \pi)\right) R}\right)|\gamma|\right) \int_{0}^{1} e^{-N \pi t / 2} d t \\
& =\frac{4\left(1-e^{-N \pi / 2}\right)}{N \pi\left(1-e^{\left.-\pi^{2} / u\right)}\right.} \exp \left(2 A+B\left(1+e^{\left(u+|\xi|^{2} /(2 \pi)\right) R}\right)|\gamma|\right) \\
& <\frac{K_{-, 3}}{N}
\end{aligned}
$$

for a positive constant $K_{-, 3}$. 
Proof of (6.4). From [1, Equation (4.6)] we have

$$
\left|I_{+, 2}(N)\right| \leq 4 e^{-2 \pi N} \int_{\varepsilon}^{1-\varepsilon}\left|g_{N}(-u /(2 \pi)+\sqrt{-1}+t)\right| d t .
$$

From 6.1) we have

$$
\begin{aligned}
& \left|g_{N}(-u /(2 \pi)+\sqrt{-1}+t)\right| \\
& \quad \leq \exp (N \operatorname{Re}(\Phi(-u /(2 \pi)+\sqrt{-1}+t))) \exp \left(4 A+2|\gamma|\left(1+e^{R\left(u+u^{2} /(2 \pi)+2 \pi\right)}\right)\right) .
\end{aligned}
$$

Now estimate $\operatorname{Re}(\Phi(-u /(2 \pi)+\sqrt{-1}+t))$. We have

$$
\begin{aligned}
& \Phi\left(-\frac{u}{2 \pi}+\sqrt{-1}+t\right) \\
= & \frac{1}{\xi}\left(\operatorname{Li}_{2}\left(e^{u+|\xi|^{2} /(2 \pi)-\xi t}\right)-\operatorname{Li}_{2}\left(e^{u-|\xi|^{2} /(2 \pi)+\xi t}\right)\right)+\frac{u^{2}}{2 \pi}-\sqrt{-1} u-u t .
\end{aligned}
$$

Since $\operatorname{Re}\left(u+|\xi|^{2} /(2 \pi)-\xi t\right)=u(1-t)+|\xi|^{2} /(2 \pi)>0$ and $\operatorname{Re}\left(u-|\xi|^{2} /(2 \pi)+\xi t\right)=$ $u(1+t)-|\xi|^{2} /(2 \pi) \leq 0$, we have

$$
\begin{aligned}
& \Phi\left(-\frac{u}{2 \pi}+\sqrt{-1}+t\right) \\
= & -\frac{1}{\xi}\left(\operatorname{Li}_{2}\left(e^{u+|\xi|^{2} /(2 \pi)-\xi t}\right)+\operatorname{Li}_{2}\left(e^{u-|\xi|^{2} /(2 \pi)+\xi t}\right)\right) \\
& -\frac{\pi^{2}}{6 \xi}-\frac{1}{2 \xi}\left(u+\frac{|\xi|^{2}}{2 \pi}-(u+2 \pi \sqrt{-1}) t+\pi \sqrt{-1}\right)^{2}+\frac{u^{2}}{2 \pi}-\sqrt{-1} u-u t
\end{aligned}
$$

from (6.12). Therefore we have

$$
\begin{aligned}
& \operatorname{Re} \Phi\left(-\frac{u}{2 \pi}+\sqrt{-1}+t\right) \\
= & -\frac{u}{|\xi|^{2}}\left(\operatorname{Re~Li}_{2}\left(e^{u+|\xi|^{2} /(2 \pi)-\xi t}\right)+\operatorname{Re}_{\operatorname{Li}_{2}}\left(e^{u-|\xi|^{2} /(2 \pi)+\xi t}\right)\right) \\
& -\frac{2 \pi}{|\xi|^{2}}\left(\operatorname{Im~Li}_{2}\left(e^{u+|\xi|^{2} /(2 \pi)-\xi t}\right)+\operatorname{Im~Li}_{2}\left(e^{u-|\xi|^{2} /(2 \pi)+\xi t}\right)\right) \\
& +(2 t-1) \pi-\frac{1}{2}\left(t^{2}+1\right) u+\frac{u^{2} t}{2 \pi}-\frac{u^{3}}{8 \pi^{2}}+\frac{\pi^{2} u}{3|\xi|^{2}} \\
\leq & -\frac{u}{|\xi|^{2}}\left(\operatorname{Re} \operatorname{Li}_{2}\left(e^{u+|\xi|^{2} /(2 \pi)-\xi t}\right)+\operatorname{Re}^{2} \operatorname{Li}_{2}\left(e^{u-|\xi|^{2} /(2 \pi)+\xi t}\right)\right) \\
& -\frac{2 \pi}{|\xi|^{2}}\left(\operatorname{Im} \operatorname{Li}_{2}\left(e^{u+|\xi|^{2} /(2 \pi)-\xi t}\right)+\operatorname{ImLi}_{2}\left(e^{u-|\xi|^{2} /(2 \pi)+\xi t}\right)\right) \\
& +(2 t-1) \pi .
\end{aligned}
$$

For $0<r<1$ and $0<\theta<2 \pi$ we have

$$
\begin{aligned}
\operatorname{Re~Li}_{2}\left(r e^{\sqrt{-1} \theta}\right) & =-\frac{1}{2} \int_{0}^{r} \frac{\log \left(1-2 s \cos \theta+s^{2}\right)}{s} d s \geq-\frac{1}{2} \int_{0}^{r} \frac{\log \left(1+2 s+s^{2}\right)}{s} d s \\
& =-\int_{0}^{r} \frac{\log (1+s)}{s} d s \geq-\int_{0}^{1} \frac{\log (1+s)}{s} d s=\operatorname{Li}_{2}(-1)=-\frac{\pi^{2}}{12} .
\end{aligned}
$$

We also have

$$
\operatorname{Im} \operatorname{Li}_{2}\left(r e^{\sqrt{-1} \theta}\right)=-\int_{0}^{r} \frac{\arg \left(1-s e^{\sqrt{-1} \theta}\right)}{s} d s
$$


If $0<\theta \leq \pi$ the right hand side is non-negative. If $\pi<\theta<2 \pi$ we have

$$
\begin{aligned}
\operatorname{Im~Li}_{2}\left(r e^{\sqrt{-1} \theta}\right) & =-\int_{0}^{1} \frac{\arg \left(1-s e^{\sqrt{-1} \theta}\right)}{s} d s+\int_{r}^{1} \frac{\arg \left(1-s e^{\sqrt{-1} \theta}\right)}{s} d s \\
& =\operatorname{ImLi}_{2}\left(e^{\sqrt{-1} \theta}\right)+\int_{r}^{1} \frac{\arg \left(1-s e^{\sqrt{-1} \theta}\right)}{s} d s .
\end{aligned}
$$

The second integral is positive and $\operatorname{Im~Li}_{2}\left(e^{\sqrt{-1} \theta}\right)$ is bigger than or equal to $-\operatorname{ImLi}_{2}(\exp (\sqrt{-1} \pi / 3))=-1.01494 \ldots$.

Therefore we have

$$
\operatorname{Re} \Phi\left(-\frac{u}{2 \pi}+\sqrt{-1}+t\right) \leq \frac{\pi^{2} u}{6|\xi|^{2}}+\frac{2 \pi \operatorname{Im~Li}_{2}\left(e^{\sqrt{-1} \pi / 3}\right)}{|\xi|^{2}}+(2 t-1) \pi
$$

So we have

$$
\begin{aligned}
& \left|I_{+, 2}(N)\right| \\
\leq & 4 \exp \left(4 A+2|\gamma|\left(1+e^{R\left(u+u^{2} /(2 \pi)+2 \pi\right)}\right)\right) e^{-3 \pi N} \\
& \times \exp \left[N\left(\frac{\pi^{2} u}{6|\xi|^{2}}+\frac{2 \pi \operatorname{Im~Li}_{2}\left(e^{\sqrt{-1} \pi / 3}\right)}{|\xi|^{2}}\right)\right] \int_{0}^{1} e^{2 N \pi t} d t \\
= & \frac{2\left(1-e^{-2 \pi N}\right)}{\pi N} \exp \left(4 A+2|\gamma|\left(1+e^{R\left(u+u^{2} /(2 \pi)+2 \pi\right)}\right)\right) \\
& \times \exp \left[\pi N\left(\frac{\pi u}{6|\xi|^{2}}+\frac{2 \operatorname{Im~Li}_{2}\left(e^{\sqrt{-1} \pi / 3}\right)}{|\xi|^{2}}-1\right)\right] \\
< & \frac{2}{\pi N} \exp \left(4 A+2|\gamma|\left(1+e^{R\left(u+u^{2} /(2 \pi)+2 \pi\right)}\right)\right) \\
< & \frac{K_{+, 2}}{N}
\end{aligned}
$$

for a positive constant $K_{+, 2}$. Here we use the inequality

$$
\frac{\pi u}{6|\xi|^{2}}+\frac{2 \operatorname{Im~Li}_{2}\left(e^{\sqrt{-1} \pi / 3}\right)}{|\xi|^{2}}-1<\frac{\pi}{6}+\frac{1.01494 \ldots}{2 \pi^{2}}-1<0 .
$$

Proof of (6.7). From [1, Equation (4.6)] we have

$$
\left|I_{-, 2}(N)\right| \leq 4 e^{-2 \pi N} \int_{\varepsilon}^{1-\varepsilon}\left|g_{N}(u /(2 \pi)-\sqrt{-1}+t)\right| d t .
$$

From (6.1) we have

$$
\begin{aligned}
& \left|g_{N}(u /(2 \pi)-\sqrt{-1}+t)\right| \\
& \quad \leq \exp (N \operatorname{Re}(\Phi(u /(2 \pi)-\sqrt{-1}+t))) \exp \left(4 A+2|\gamma|\left(1+e^{R\left(u+u^{2} /(2 \pi)+2 \pi\right)}\right)\right) .
\end{aligned}
$$

Now estimate $\operatorname{Re}(\Phi(u /(2 \pi)-\sqrt{-1}+t))$. We have

$$
\begin{aligned}
& \Phi\left(\frac{u}{2 \pi}-\sqrt{-1}+t\right) \\
= & \frac{1}{\xi}\left(\operatorname{Li}_{2}\left(e^{u-|\xi|^{2} /(2 \pi)-\xi t}\right)-\operatorname{Li}_{2}\left(e^{u+|\xi|^{2} /(2 \pi)+\xi t}\right)\right)-\frac{u^{2}}{2 \pi}+\sqrt{-1} u-u t .
\end{aligned}
$$


Since $\operatorname{Re}\left(u-|\xi|^{2} /(2 \pi)-\xi t\right)=u(1-t)-|\xi|^{2} /(2 \pi)<0$ and $\operatorname{Re}\left(u+|\xi|^{2} /(2 \pi)+\xi t\right)=$ $u(1+t)+|\xi|^{2} /(2 \pi)>0$, we have

$$
\begin{aligned}
& \Phi\left(\frac{u}{2 \pi}-\sqrt{-1}+t\right) \\
= & \frac{1}{\xi}\left(\operatorname{Li}_{2}\left(e^{u-|\xi|^{2} /(2 \pi)-\xi t}\right)+\operatorname{Li}_{2}\left(e^{-u-|\xi|^{2} /(2 \pi)-\xi t}\right)\right) \\
& +\frac{\pi^{2}}{6 \xi}+\frac{1}{2 \xi}\left(u+\frac{|\xi|^{2}}{2 \pi}+(u+2 \pi \sqrt{-1}) t-\pi \sqrt{-1}\right)^{2}-\frac{u^{2}}{2 \pi}+\sqrt{-1} u-u t
\end{aligned}
$$

from 6.12). Therefore we have

$$
\begin{aligned}
& \operatorname{Re} \Phi\left(\frac{u}{2 \pi}-\sqrt{-1}+t\right) \\
& =\frac{u}{|\xi|^{2}}\left(\operatorname{ReLi}_{2}\left(e^{u-|\xi|^{2} /(2 \pi)-\xi t}\right)+\operatorname{Re}^{2} \operatorname{Li}_{2}\left(e^{-u-|\xi|^{2} /(2 \pi)-\xi t}\right)\right) \\
& +\frac{2 \pi}{|\xi|^{2}}\left(\operatorname{Im~Li}_{2}\left(e^{u-|\xi|^{2} /(2 \pi)-\xi t}\right)+\operatorname{ImLi}_{2}\left(e^{-u-|\xi|^{2} /(2 \pi)-\xi t}\right)\right) \\
& +\frac{u \pi^{2}}{6|\xi|^{2}}-\frac{u^{2}}{2 \pi}-u t \\
& +\frac{u}{2|\xi|^{2}}\left(\left(u+\frac{|\xi|^{2}}{2 \pi}+u t\right)^{2}-\pi^{2}(2 t-1)^{2}\right) \\
& +\frac{4 \pi}{2|\xi|^{2}} \pi(2 t-1)\left(u+\frac{|\xi|^{2}}{2 \pi}+u t\right) \\
& =\frac{u}{|\xi|^{2}}\left(\operatorname{ReLi}_{2}\left(e^{u-|\xi|^{2} /(2 \pi)-\xi t}\right)+\operatorname{Re}^{2} \operatorname{Li}_{2}\left(e^{-u-|\xi|^{2} /(2 \pi)-\xi t}\right)\right) \\
& +\frac{2 \pi}{|\xi|^{2}}\left(\operatorname{Im~Li}_{2}\left(e^{u-|\xi|^{2} /(2 \pi)-\xi t}\right)+\operatorname{ImLi}_{2}\left(e^{-u-|\xi|^{2} /(2 \pi)-\xi t}\right)\right) \\
& +\frac{u t^{2}}{2}+\frac{|\xi|^{2} t}{2 \pi}-\frac{7 u \pi^{2}}{3|\xi|^{2}}+\frac{u^{3}}{2|\xi|^{2}}+\frac{u|\xi|^{2}}{8 \pi^{2}}-\pi .
\end{aligned}
$$

For $0<r<1$ and $0<\theta<2 \pi$ we have

$$
\begin{aligned}
\operatorname{Re~Li}_{2}\left(r e^{\sqrt{-1} \theta}\right) & =-\frac{1}{2} \int_{0}^{r} \frac{\log \left(1-2 s \cos \theta+s^{2}\right)}{s} d s \leq-\frac{1}{2} \int_{0}^{r} \frac{\log \left(1-2 s+s^{2}\right)}{s} d s \\
& =-\int_{0}^{r} \frac{\log (1-s)}{s} d s \leq-\int_{0}^{1} \frac{\log (1-s)}{s} d s=\operatorname{Li}_{2}(1)=\frac{\pi^{2}}{6} .
\end{aligned}
$$

We also have

$$
\begin{aligned}
\operatorname{Im} \operatorname{Li}_{2}\left(r e^{\sqrt{-1} \theta}\right) & =-\int_{0}^{r} \frac{\arg \left(1-s e^{\sqrt{-1} \theta}\right)}{s} d s \\
& =-\int_{0}^{1} \frac{\arg \left(1-s e^{\sqrt{-1} \theta}\right)}{s} d s+\int_{r}^{1} \frac{\arg \left(1-s e^{\sqrt{-1} \theta}\right)}{s} d s \\
& \leq \operatorname{Im} \operatorname{Li}_{2}\left(e^{\sqrt{-1} \theta}\right) \leq \operatorname{Im~Li}_{2}\left(e^{\sqrt{-1} \pi / 3}\right)=1.01494 \ldots
\end{aligned}
$$


when $0 \leq \theta \leq \pi$. Since $\operatorname{ImLi}_{2}\left(r e^{\sqrt{-1} \theta}\right)=-\operatorname{ImLi}_{2}\left(r e^{\sqrt{-1}(\theta-\pi)}\right)$ when $\pi<\theta \leq 2 \pi$, we have

$$
\begin{aligned}
& \operatorname{Re} \Phi\left(\frac{u}{2 \pi}-\sqrt{-1}+t\right) \\
& =\frac{4 \pi}{|\xi|^{2}} \operatorname{Im} \operatorname{Li}_{2}\left(e^{\sqrt{-1} \pi / 3}\right)+\frac{u t^{2}}{2}+\frac{|\xi|^{2} t}{2 \pi}-2 \frac{u \pi^{2}}{|\xi|^{2}}+\frac{u^{3}}{2|\xi|^{2}}+\frac{u|\xi|^{2}}{8 \pi^{2}}-\pi \\
& \text { (since } 0<t<1 \text { and } 0<u<\pi / 2 \text { ). } \\
& <\frac{|\xi|^{2} t}{2 \pi}+\frac{1}{\pi} \operatorname{Im~Li}_{2}\left(e^{\sqrt{-1} \pi / 3}\right)+\frac{\pi}{2}-\frac{4 \pi}{17}+\frac{\pi}{64}+\frac{17 \pi}{64}-\pi \\
& \leq \frac{|\xi|^{2} t}{2 \pi}+\frac{1}{\pi} \operatorname{Im~Li}_{2}\left(e^{\sqrt{-1} \pi / 3}\right)-\frac{247}{544} \pi .
\end{aligned}
$$

when $u<\pi / 2$.

So we have

$$
\begin{aligned}
& \left|I_{-, 2}(N)\right| \\
< & 4 e^{\left(\operatorname{Im~Li}_{2}\left(e^{\sqrt{-1} \pi / 3}\right) / \pi-247 \pi / 544-2 \pi\right) N} \exp \left(4 A+2|\gamma|\left(1+e^{R\left(u+u^{2} /(2 \pi)+2 \pi\right)}\right)\right) \\
& \times \lim _{\varepsilon \searrow 0} \int_{\varepsilon}^{1-\varepsilon} e^{N|\xi|^{2} t /(2 \pi)} d t \\
= & 4 e^{\left(\operatorname{Im~Li}_{2}\left(e^{\sqrt{-1} \pi / 3}\right) / \pi-247 \pi / 544-2 \pi\right) N} \exp \left(4 A+2|\gamma|\left(1+e^{R\left(u+u^{2} /(2 \pi)+2 \pi\right)}\right)\right) \\
& \times \frac{2 \pi}{N|\xi|^{2}}\left(e^{N|\xi|^{2} /(2 \pi)}-1\right) \\
= & \frac{8 \pi \exp \left(4 A+2|\gamma|\left(1+e^{R\left(u+u^{2} /(2 \pi)+2 \pi\right)}\right)\right)}{N|\xi|^{2}} \\
& \times\left(e^{\left(\operatorname{Im} \operatorname{Li}_{2}\left(e^{\sqrt{-1} \pi / 3}\right) / \pi-247 \pi / 544-2 \pi+|\xi|^{2} /(2 \pi)\right) N}\right. \\
< & \frac{8 \pi \exp \left(4 A+2|\gamma|\left(1+e^{R\left(u+u^{2} /(2 \pi)+2 \pi\right)}\right)\right)}{N|\xi|^{2}} \\
& \times\left(e^{\left(\operatorname{Im} \operatorname{Li}_{2}\left(e^{\sqrt{-1} \pi / 3}\right) / \pi-179 \pi / 544\right) N}-e^{\left(\operatorname{Im} \operatorname{Li}_{2}\left(e^{\sqrt{-1} \pi / 3}\right) / \pi-247 \pi / 544-2 \pi\right) N}\right) \\
< & \frac{8 \pi \exp \left(4 A+2|\gamma|\left(1+e^{R\left(u+u^{2} /(2 \pi)+2 \pi\right)}\right)\right)}{N|\xi|^{2}} \\
<-, 2 & \frac{K}{N}
\end{aligned}
$$

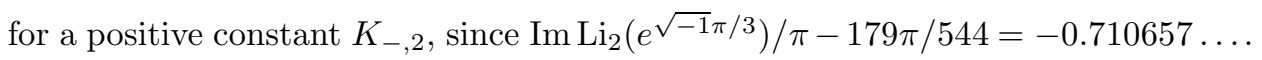

\section{Proof of Proposition 3.2}

In this section we again follow [1] to prove Proposition 3.2, From (6.1) we have

$$
\int_{p(\varepsilon)} g_{N}(w) d w=\int_{p(\varepsilon)} \exp (N \Phi(w)) d w+\int_{p(\varepsilon)} \exp (N \Phi(w)) h_{\gamma}(w) d w
$$

for any path in the parallelogram bounded by $C_{+}(\varepsilon) \cup C_{-}(\varepsilon)$ connecting $\varepsilon$ and $1-\varepsilon$, where

$$
h_{\gamma}(w):=\exp \left(I_{\gamma}(\pi-\sqrt{-1} u+\sqrt{-1} \xi w)-I_{\gamma}(-\pi-\sqrt{-1} u-\sqrt{-1} \xi w)\right)-1 .
$$

Note that $h_{\gamma}(w)$ is defined for $w$ with $0<\operatorname{Im}(\xi w)<2 \pi$, that is, for $w$ between the two parallel thick lines depicted in Figure 1 . 
Then we have

$$
\begin{aligned}
& \left|\int_{p(\varepsilon)} g_{N}(w) d w-\int_{p(\varepsilon)} \exp (N \Phi(w)) d w\right| \\
\leq & \int_{p(\varepsilon)}\left|\exp (N \Phi(w)) h_{\gamma}(w)\right| d w \\
\leq & \max _{w \in p(\varepsilon)}\{\exp (N \operatorname{Re} \Phi(w))\} \int_{p(\varepsilon)}\left|h_{\gamma}(w)\right| d w \\
= & \max _{w \in p(\varepsilon)}\{\exp (N \operatorname{Re} \Phi(w))\} \int_{\varepsilon}^{1-\varepsilon}\left|h_{\gamma}(w)\right| d w \\
\leq & \max _{w \in p(\varepsilon)}\{\exp (N \operatorname{Re} \Phi(w))\} \int_{0}^{1}\left|h_{\gamma}(w)\right| d w .
\end{aligned}
$$

Here we use the analyticity of $h_{\gamma}$ in the equality.

By the definition of $h_{\gamma}$ we have

$$
h_{\gamma}(t)=\sum_{n=1}^{\infty} \frac{1}{n !}\left(I_{\gamma}(\pi-\sqrt{-1} u+\sqrt{-1} \xi t)-I_{\gamma}(-\pi-\sqrt{-1} u-\sqrt{-1} \xi t)\right)^{n} .
$$

From Lemma 6.1, for $0<t<1$ we have

$$
\left|I_{\gamma}(\pi-\sqrt{-1} u+\sqrt{-1} \xi t)\right| \leq A|\gamma|\left(\frac{1}{2 \pi t}+\frac{1}{2 \pi-2 \pi t}\right)+B|\gamma|\left(1+e^{(u-u t) R}\right)
$$

and

$$
\left|I_{\gamma}(-\pi-\sqrt{-1} u-\sqrt{-1} \xi w)\right| \leq A|\gamma|\left(\frac{1}{2 \pi-2 \pi t}+\frac{1}{2 \pi t}\right)+B|\gamma|\left(1+e^{(u+u t) R}\right),
$$

and so we have

$$
\begin{aligned}
& \left|I_{\gamma}(\pi-\sqrt{-1} u+2 \pi t)-I_{\gamma}(-\pi-\sqrt{-1} u-2 \pi t)\right| \\
\leq & \frac{A|\gamma|}{\pi}\left(\frac{1}{1-t}+\frac{1}{t}\right)+B|\gamma|\left(2+e^{(1+t) u R}+e^{(1-t) u R}\right) \\
\leq & |\gamma|\left(A^{\prime} f(t)+B^{\prime}\right)
\end{aligned}
$$

for some positive constants $A^{\prime}$ and $B^{\prime}$, where we put $f(t):=1 / t+1 /(1-t)$. Since $f(t) \geq 4$ for $0<t<1$ we have

$$
\begin{aligned}
& \left|I_{\gamma}(\pi-\sqrt{-1} u+\sqrt{-1} \xi w)-I_{\gamma}(-\pi-\sqrt{-1} u-\sqrt{-1} \xi w)\right| \\
\leq & |\gamma|\left(A^{\prime} f(t)+B^{\prime} \frac{f(t)}{4}\right) \\
= & A^{\prime \prime}|\gamma| f(t),
\end{aligned}
$$

where $A^{\prime \prime}:=A^{\prime}+B^{\prime} / 4$.

From the argument in [1, P. 537] we have

$$
\int_{|\gamma|}^{1-|\gamma|} f(t)^{n} d t \leq 2^{2 n+1} \int_{|\gamma|}^{1 / 2} \frac{d t}{t^{n}}
$$

for $n \geq 1$. Since $|\gamma|=|\xi| /(2 N)$ we have

$$
\int_{|\gamma|}^{1 / 2} \frac{d t}{t}=\log N-\log |\xi| \leq \log N
$$

and

for $n \geq 2$.

$$
\int_{|\gamma|}^{1 / 2} \frac{d t}{t^{n}}=\frac{1}{n-1}\left(\frac{1}{|\gamma|^{n-1}}-2^{n-1}\right) \leq \frac{1}{(n-1)|\gamma|^{n-1}}
$$


Therefore from (7.2) and (7.3) we have

$$
\begin{aligned}
\int_{|\gamma|}^{1-|\gamma|}\left|h_{\gamma}(t)\right| d t & \leq \sum_{n=1}^{\infty} \frac{1}{n !}\left(A^{\prime \prime}\right)^{n}|\gamma|^{n} \int_{|\gamma|}^{1-|\gamma|} f(t)^{n} d t \\
& \leq 2|\gamma|\left(4 A^{\prime \prime} \log N+\sum_{n=2}^{\infty} \frac{\left(4 A^{\prime \prime}\right)^{n}}{(n-1) n !}\right) \\
& \leq \frac{|\xi|}{N}\left(4 A^{\prime \prime} \log N+\exp \left(4 A^{\prime \prime}\right)-4 A^{\prime \prime}-1\right) \\
& \leq \frac{K^{\prime} \log N}{N}
\end{aligned}
$$

for a positive constant $K^{\prime}$ if $N$ is sufficiently large.

For $0 \leq t \leq 1$, we also have from Lemma 6.1

$$
\begin{aligned}
& \left|I_{\gamma}(\pi-\sqrt{-1} u+2 \pi t)-I_{\gamma}(-\pi-\sqrt{-1} u-2 \pi t)\right| \\
\leq & 4 A+B|\gamma|\left(2+e^{(1+t) u R}+e^{(1-t) u R}\right) .
\end{aligned}
$$

Since $|\gamma|=|\xi| /(2 N)$, we have

$$
\left|h_{\gamma}(t)\right| \leq \exp \left(4 A+\frac{B^{\prime}|\xi|}{2 N}\right)
$$

So we have

$$
\int_{0}^{|\gamma|}\left|h_{\gamma}(t)\right| d t \leq \frac{|\xi|}{2 N} \exp \left(4 A+\frac{B^{\prime}|\xi|}{2 N}\right) \leq \frac{K^{\prime \prime}}{N}
$$

and

$$
\int_{1-|\gamma|}^{1}\left|h_{\gamma}(t)\right| d t \leq \frac{|\xi|}{2 N} \exp \left(4 A+\frac{B^{\prime}|\xi|}{2 N}\right) \leq \frac{K^{\prime \prime \prime}}{N}
$$

for positive constants $K^{\prime \prime}$ and $K^{\prime \prime \prime}$.

Therefore we have

$$
\int_{0}^{1}\left|h_{\gamma}(t)\right| d t \leq \frac{K^{\prime} \log N}{N}+\frac{K^{\prime \prime}}{N}+\frac{K^{\prime \prime \prime}}{N} \leq \frac{K_{2} \log N}{N}
$$

for a positive constant $K_{2}$. Now from (7.1) we finally have

$$
\left|\int_{p(\varepsilon)} g_{N}(w) d w-\int_{p(\varepsilon)} \exp (N \Phi(w)) d w\right| \leq \frac{K_{2} \log N}{N} \max _{w \in p(\varepsilon)}\{\exp (N \operatorname{Re} \Phi(w))\}
$$

proving Proposition 3.2 .

\section{Proof of Lemma 3.4}

We will show that $\xi \Phi\left(w_{0}\right)$ is purely imaginary with positive imaginary part. Then since $\xi$ is in the first quadrant, $\Phi\left(w_{0}\right)$ is in also in the first quadrant and so $\operatorname{Re} \Phi\left(w_{0}\right)>0$.

Since $\varphi(u)$ is purely imaginary (Remark [3.3), we have $\operatorname{Li}_{2}\left(e^{u-\varphi(u)}\right)=$ $\overline{\mathrm{Li}_{2}\left(e^{u+\varphi(u)}\right)}$. Therefore from (3.4) and (6.12) we have

$$
\begin{aligned}
\operatorname{Im}\left(\xi \Phi\left(w_{0}\right)\right) & =-2 \operatorname{Im}_{L_{2}\left(e^{u+\varphi(u)}\right)-u \operatorname{Im} \tilde{\varphi}(u)} \\
& =2 \operatorname{Im} \operatorname{Li}_{2}\left(e^{-u-\varphi(u)}\right)+\operatorname{Im}(u+\varphi(u)+\pi \sqrt{-1})^{2}-u \operatorname{Im} \tilde{\varphi}(u) \\
& =2 \operatorname{Im}_{L_{2}}\left(e^{-u-\varphi(u)}\right)+u \varphi(u) .
\end{aligned}
$$


So we have

$$
\begin{aligned}
& \frac{d \operatorname{Im}\left(\xi \Phi\left(w_{0}\right)\right)}{d u} \\
&= 2 \operatorname{Im}\left(\log \left(1-e^{-u-\varphi(u)}\right)\left(1+\sqrt{-1} \frac{d \operatorname{Im} \varphi(u)}{d u}\right)\right)+\operatorname{Im} \varphi(u)+u \frac{d \operatorname{Im} \varphi(u)}{d u} \\
&= \frac{d \operatorname{Im} \varphi(u)}{d u}\left(2 \log \left|1-e^{-u-\varphi(u)}\right|+u\right)+2 \arg \left(1-e^{-u-\varphi(u)}\right)+\operatorname{Im} \varphi(u) \\
&= \frac{d \operatorname{Im} \varphi(u)}{d u} \log \left(\left(1-e^{-u-\varphi(u)}\right)\left(1-e^{-u+\varphi(u)}\right) e^{u}\right)+2 \arg \left(1-e^{-u-\varphi(u)}\right)+\operatorname{Im} \varphi(u) \\
&= \frac{d \operatorname{Im} \varphi(u)}{d u} \log \left(e^{u}+e^{-u}-e^{\varphi(u)}-e^{-\varphi(u)}\right)+2 \arg \left(1-e^{-u-\varphi(u)}\right)+\operatorname{Im} \varphi(u) \\
&= 2 \arg \left(1-e^{-u-\varphi(u)}\right)+\operatorname{Im} \varphi(u)<0 \\
& \text { since }-\pi / 3<\operatorname{Im} \varphi(u)<0 .
\end{aligned}
$$

Since $\varphi(0)=-\pi \sqrt{-1} / 3$ and $\varphi(\log ((3+\sqrt{5}) / 2))=0$, we have

$$
\xi \Phi\left(w_{0}\right)= \begin{cases}2 \operatorname{ImLi}_{2}\left(e^{\pi \sqrt{-1} / 3}\right)=1.01494 \ldots & (u=0), \\ 2 \operatorname{ImLi}_{2}\left(\frac{2}{3+\sqrt{5}}\right)=0 & (u=\log ((3+\sqrt{5}) / 2)) .\end{cases}
$$

Therefore $\operatorname{Im}\left(\xi \Phi\left(w_{0}\right)\right)>0$ for $0<u<\log ((3+\sqrt{5}) / 2)$, completing the proof of Lemma 3.4

\section{REFERENCES}

[1] J. E. Andersen and S. K. Hansen, Asymptotics of the quantum invariants for surgeries on the figure 8 knot, J. Knot Theory Ramifications 15 (2006), no. 4, 479-548. MR MR2221531

[2] T. Dimofte and S. Gukov, Quantum field theory and the volume conjecture, to appear in Contemporary Mathematics "Interactions Between Hyperbolic Geometry, Quantum Topology and Number Theory", arXiv:1003.4808, 2010.

[3] T. Dimofte, S. Gukov, J. Lenells, and D. Zagier, Exact results for perturbative Chern-Simons theory with complex gauge group, Commun. Number Theory Phys. 3 (2009), no. 2, 363-443. MR 2551896 (2010k:58038)

[4] J. Dubois, Non abelian twisted Reidemeister torsion for fibered knots, Canad. Math. Bull. 49 (2006), no. 1, 55-71. MR MR2198719

[5] J. Dubois and R. M. Kashaev, On the asymptotic expansion of the colored jones polynomial for torus knots, Math. Ann. 339 (2007), no. 4, 757-782.

[6] L. D. Faddeev, Discrete Heisenberg-Weyl group and modular group, Lett. Math. Phys. 34 (1995), no. 3, 249-254. MR 1345554 (96i:46075)

[7] S. Gukov, Three-dimensional quantum gravity, Chern-Simons theory, and the A-polynomial, Comm. Math. Phys. 255 (2005), no. 3, 577-627. MR MR2134725

[8] S. Gukov and H. Murakami, SL $(2, \mathbf{C})$ Chern-Simons theory and the asymptotic behavior of the colored Jones polynomial, Modular Forms and String Duality (N. Yui, H. Verrill, and C.F. Doran, eds.), Fields Inst. Comm., vol. 54, Amer. Math. Soc. and Fields Inst., 2008, pp. 261-278.

[9] K. Hikami and H. Murakami, Representations and the colored jones polynomial of a torus knot, to appear in AMS/IP Studies in Advanced Mathematics "Chern-Simons Theory: 20 years after", arXiv:1001.2680, 2010.

[10] H. Hilden, M. Lozano, and J. M. Montesinos-Amilibia, On a remarkable polyhedron geometrizing the figure eight knot cone manifolds, J. Math. Sci. Univ. Tokyo 2 (1995), no. 3, 501-561. MR MR1382519 (96m:57021)

[11] V. F. R. Jones, A polynomial invariant for knots via von Neumann algebras, Bull. Amer. Math. Soc. (N.S.) 12 (1985), no. 1, 103-111. MR 86e:57006

[12] R. M. Kashaev, A link invariant from quantum dilogarithm, Modern Phys. Lett. A 10 (1995), no. 19, 1409-1418. MR 96j:81060

[13] _ The hyperbolic volume of knots from the quantum dilogarithm, Lett. Math. Phys. 39 (1997), no. 3, 269-275. MR 98b:57012 
[14] P. Kirk and E. Klassen, Chern-Simons invariants of 3-manifolds decomposed along tori and the circle bundle over the representation space of $T^{2}$, Comm. Math. Phys. 153 (1993), no. 3, 521-557. MR 94d:57042

[15] E. P. Klassen, Representations of knot groups in SU(2), Trans. Amer. Math. Soc. 326 (1991), no. 2, 795-828. MR MR1008696 (91k:57003)

[16] J. E. Marsden and M. J. Hoffman, Basic complex analysis, W. H. Freeman and Company, New York, 1987. MR 88m:30001

[17] G. Masbaum, Skein-theoretical derivation of some formulas of Habiro, Algebr. Geom. Topol. 3 (2003), 537-556 (electronic). MR MR1997328 (2004f:57013)

[18] V. Muñoz, The SL(2, C)-character varieties of torus knots, Rev. Mat. Complut. 22 (2009), no. 2, 489-497. MR MR2553945

[19] H. Murakami, An introduction to the volume conjecture and its generalizations, Acta Math. Vietnam. 33 (2008), no. 3, 219-253. MR MR2501844

[20] _ An introduction to the volume conjecture, to appear in Contemporary Mathematics "Interactions Between Hyperbolic Geometry, Quantum Topology and Number Theory", arXiv:1002:0126, 2010.

[21] H. Murakami and J. Murakami, The colored Jones polynomials and the simplicial volume of a knot, Acta Math. 186 (2001), no. 1, 85-104. MR 2002b:57005

[22] H. Murakami and Y. Yokota, The colored Jones polynomials of the figure-eight knot and its Dehn surgery spaces, J. Reine Angew. Math. 607 (2007), 47-68. MR MR2338120

[23] W. D. Neumann and D. Zagier, Volumes of hyperbolic three-manifolds, Topology 24 (1985), no. 3, 307-332. MR 87j:57008

[24] J. Porti, Torsion de Reidemeister pour les variétés hyperboliques, Mem. Amer. Math. Soc. 128 (1997), no. 612, x+139. MR MR1396960 (98g:57034)

[25] R. Riley, Nonabelian representations of 2-bridge knot groups, Quart. J. Math. Oxford Ser. (2) 35 (1984), no. 138, 191-208. MR MR745421 (85i:20043)

[26] W. P. Thurston, The Geometry and Topology of Three-Manifolds, Electronic version 1.1 March 2002, http://www.msri.org/publications/books/gt3m/.

[27] T. Yoshida, The $\eta$-invariant of hyperbolic 3-manifolds, Invent. Math. 81 (1985), no. 3, 473514. MR 87f:58153

Department of Mathematics, Tokyo Institute of Technology, Oh-okayama, Meguro, TOKYO 152-8551, JAPAN

E-mail address: starshea@tky3.3web.ne.jp 